\title{
Quantitative Evidence for Direct Effects Between Earth-Ionosphere Schumann Resonances and Human Cerebral Cortical Activity
}

\author{
Kevin S. Saroka ${ }^{a}$, Michael A. Persinger ${ }^{b}$ \\ Laurentian University, Sudbury, Ontario, P32 2C6, Canada \\ ${ }^{a, b}$ E-mail addresses: kx_saroka@laurentian.ca ; mpersinger@laurentian.ca
}

\begin{abstract}
The multiple quantitative similarities of basic frequencies, harmonics, magnetic field intensities, voltages, band widths, and energetic solutions that define the Schumann resonances within the separation between the earth and ionosphere and the activity within the human cerebral cortices suggest the capacity for direct interaction. The recent experimental demonstration of the representations of the Schumann resonances within the spectral densities of normal human quantitative electroencephalographic (QEEG) activity suggests a casual interaction. Calculations supported by correlations between amplitudes of the global Schumann resonances measured several thousands of $\mathrm{km}$ away (which were nearly identical to our local measurements) and the coherence and current densities or these frequency bands between cerebral hemispheres for a large population of human QEEG measures indicate that such interaction occurs. The energies are within the range that would allow information to be exchanged between cerebral and Schumann sources. The near-identical solution for current density from the increasing human population and background vertical electric fields suggests that changes in the former might determine the degree of coherence between the Schumann resonances. Direct comparisons of local Schumann measurements and brain activity exhibited powerful intermittent coherence within the first three harmonics. Implications of the contributions of solar transients, surface temperature, and rapidly developing technologies to modify the ionosphere's Schumann properties are considered.
\end{abstract}

Keywords: Schumann harmonics; quantitative electroencephalography; brain-ionosphere similarities; picoTesla intensities; correlations

\section{INTRODUCTION}

When two systems share remarkably similar physical characteristics such as dynamic amplitudes and intrinsic frequencies the possibility for direct interaction emerges. This assumes impedances and reluctances match or shared resonance occurs between the electromagnetic energies. The thin shell between the ionosphere and earth generates continuous harmonics of frequencies from a fundamental of about 7 to $8 \mathrm{~Hz}$ that is caused by global lightning which occurs between 40 to 100 times per second (40 to $100 \mathrm{~Hz}$ ). These frequencies are the Schumann resonances. 
The bulk velocity of neuronal activity around the human cerebral cortices caused by discharge of action potentials within this thin shell of tissue generates a resonance with a fundamental between 7 to $8 \mathrm{~Hz}$. On the bases of the average durations of the travelling waves over the cerebral cortices the repetition rate and phase velocities are in the order of 40 to 80 times per second (40 to $80 \mathrm{~Hz}$ ). The current density around the annulus of an axon associated with a single action potential is equivalent to about $10^{5} \mathrm{~A} \cdot \mathrm{m}^{-1}$ or the value associated with a single lightning discharge [1].

For both cerebral and earth-ionosphere phenomena the average potential difference for these time-varying processes is in the range of $0.5 \mathrm{mV} \cdot \mathrm{m}^{-1}$. The magnetic field component is the order of $2 \mathrm{pT}\left(10^{-12} \mathrm{~T}\right)$. The ratio of this voltage gradient to the magnetic field intensity is effectively the velocity of light, $\sim 3 \cdot 10^{8} \mathrm{~m} \cdot \mathrm{s}^{-1}$. These general similarities suggest that more detailed congruence could occur. Here we present quantitative evidence that the electric and magnetic fields of the Schumann resonances and those equivalent frequencies generated by the human cerebral cortices can interact and may be in a persistent state of equilibrium.

\section{BASIC FEATURES OF SCHUMANN FREQUENCIES}

A recent discussion [2] of the Schumann Resonance characteristics summarizes the information found within Nickolaenko and Hayakawa [3], König et al [4] and Cherry [5]. Briefly, the harmonics or modes of the Schumann resonances peak around $7.8 \mathrm{~Hz}, 14.1 \mathrm{~Hz}$, $20.3 \mathrm{~Hz}, 26.4 \mathrm{~Hz}$, and $32.5 \mathrm{~Hz}$. This serial shift of about $6 \mathrm{~Hz}$ is consistent with the relation of:

$$
[\sqrt{ }(\mathrm{n}(\mathrm{n}+1))] \cdot\left[\left(\mathrm{v} \cdot(2 \pi \mathrm{r})^{-1}\right]\right.
$$

where $\mathrm{n}$ are serial integers $\geq 1, \mathrm{v}$ is the velocity of light in the medium (which is effectively $\mathrm{c}$ ) and $r$ is the radius of the earth. The first component of the relation when " $n$ " is substituted as a quantum number is also employed to calculate the magnitude of the orbital angular momentum [6]. When magnetic moments are expressed as Bohr magnetons the electron state is associated with a magnetic moment equal to $\sqrt{ }(n(n+1))$. Changes in electron states or different shells are associated with emissions of photons.

The peak values are not precise and can vary by $\pm 0.2 \mathrm{~Hz}$ depending upon ionosphericearth conditions, time of day, season, influx of protons from solar events, pre-earthquake conditions, and yet to be identified sources. According to Nickolaenko and Hayakawa [3] monthly variations in the first mode $(7.8 \mathrm{~Hz}$ ) range between 7.8 and $8.0 \mathrm{~Hz}$. The diurnal variation in frequency shift has been attributed to drifts in global lightning or alterations in ionospheric height. Peak frequencies and amplitudes occur in May whereas minima occur in October-November. The peak to peak modulations are about $\pm 25 \%$ of the median value.

The optimal metaphor is that every lighting strike of the approximately 40 to 100 per second between the ionosphere and ground is an expanding wave that moves until it ultimately interfaces with itself on the earth's spherical surface. The resulting interface elicits a return wave that arrives at the original source within about $125 \mathrm{~ms}$ after the initiation. The approximate equivalent frequency is $8 \mathrm{~Hz}$. The attenuation is minimal over megameters.

Resonance amplitudes are remarkably similar even when recorded simultaneously at observatories separated by large distances and reflect the variations in global thunderstorm activity. The maximum intensities, displayed by the first and second harmonic, are $\sim 3 \mathrm{pT}$ which diminish to $\sim 1 \mathrm{pT}$ around $20.3 \mathrm{~Hz}$ and less than $0.5 \mathrm{pT}$ for the higher harmonics. The 
vertical electric (E) field strengths display ranges in the order of 0.3 to $1.0 \mathrm{mV} \cdot \mathrm{m}^{-1}$. The coherence of the E field strength for the first harmonic is about 0.8 to 0.9 over thousands of $\mathrm{km}$.

To discern the Schumann spectral densities continuous measurements between 10 and $100 \mathrm{~s}$ are required. This is similar to the optimal sampling for clear resolution for spectral densities of human cerebral activity. With $10 \mathrm{~s}$ and $100 \mathrm{~s}$ samples the spectral resolutions are between $0.1 \mathrm{~Hz}$ and $0.01 \mathrm{~Hz}$, respectively. For discerning the relationship between phase shift and frequency at various stations record lengths are about $10 \mathrm{~min}$.

The first or fundamental resonance "splits" into three subfrequencies because of the different phase velocities and attenuation rates corresponding to standing waves and those moving in either an east-west or west-east direction. Because the peak width of the fundamental or base frequency is $2 \mathrm{~Hz}(7$ to $9 \mathrm{~Hz})$ and separation is in the order of a few 0.1 $\mathrm{Hz}$, discrimination has been a technical challenge. A frequency shift between $7 \mathrm{~Hz}$ and $9 \mathrm{~Hz}$ with $8 \mathrm{~Hz}$ as the reference involves a phase shift between $-20^{\circ}$ and $+20^{\circ}$.

The Schumann resonances are subject to modification by imminent seismological and thermal energies. Different amplitudes of the fourth harmonic, concurrent with a shift as much as $1 \mathrm{~Hz}$, can appear approximately one week before an earthquake. The modification remains for days and can still be present during aftershocks [3]. For every $1 \mathrm{deg} \mathrm{C}$ increase in tropical temperature the magnetic field measured increases by about $3 \mu \mathrm{A} \cdot \mathrm{m}^{-1}$ or $3.8 \mathrm{pT}$. Recently Streltsov et al [7] experimentally doubled the amplitude of the $8 \mathrm{~Hz}$ E field of the Schumann resonance by focusing packets of energy into the cavity that matched the second to third gyroharmonic electromagnetic wave ( $\mathrm{MHz}$ range) values of electrons.

The spherical cavity of the earth-ionosphere that "traps the Schumann resonances" exhibits Eigen values that can be characterized by the first three equivalents of the quantum numbers: $\mathrm{p}, \mathrm{n}$, and $\mathrm{m}$. $\mathrm{P}$ indicates how many half-wavelengths can fit the ionospheric height which reflect the Eigen-values or resonance frequencies. N (zonal number) describes how many wavelengths fit around the circumference of the earth. $M$, the azimunthal number, reflects the numbers of wavelengths along the equator moving from east to west or west to east [3].

These descriptors are similar to the four quantum numbers that have been employed to describe the primary dynamic features of the atom that is the electron. These are mentioned from the perspective of integrating physics, chemistry and astronomy within the interdisciplinary framework. The similarity may also support the traditional philosophical concept that microcosm reflects macrocosm.

\section{BASIC FEATURES OF THE DYNAMIC HUMAN BRAIN}

The human cerebrum $(1.35 \mathrm{~kg})$ is an ellipsoid aggregate of matter that occupies about $1.3 \cdot 10^{-3} \mathrm{~m}^{3}$. The three-dimensional metrics [8] are: length $(155-190 \mathrm{~mm})$, width $(131-141$ $\mathrm{mm})$, depth $(108-117 \mathrm{~mm})$. The cerebral cortices are approximately 1 to $4 \mathrm{~mm}$ thick but occupy almost $40 \%$ of the cerebral volume with an average value of $490 \mathrm{cc}$ [9]. The surface area of the human brain is not smooth but exhibits convexities (gyri) and concavities (sulci).

Two-thirds of the surface is buried within the sulci. Mathematical modeling indicates that this topological surface is similar to that of a flat surface "wrinkled" into the third dimension. Secondary extrusions with widths of about $1 \mathrm{~mm}$ emerge intermittently along the trough of the sulci and the apex of the gyri [10]. 


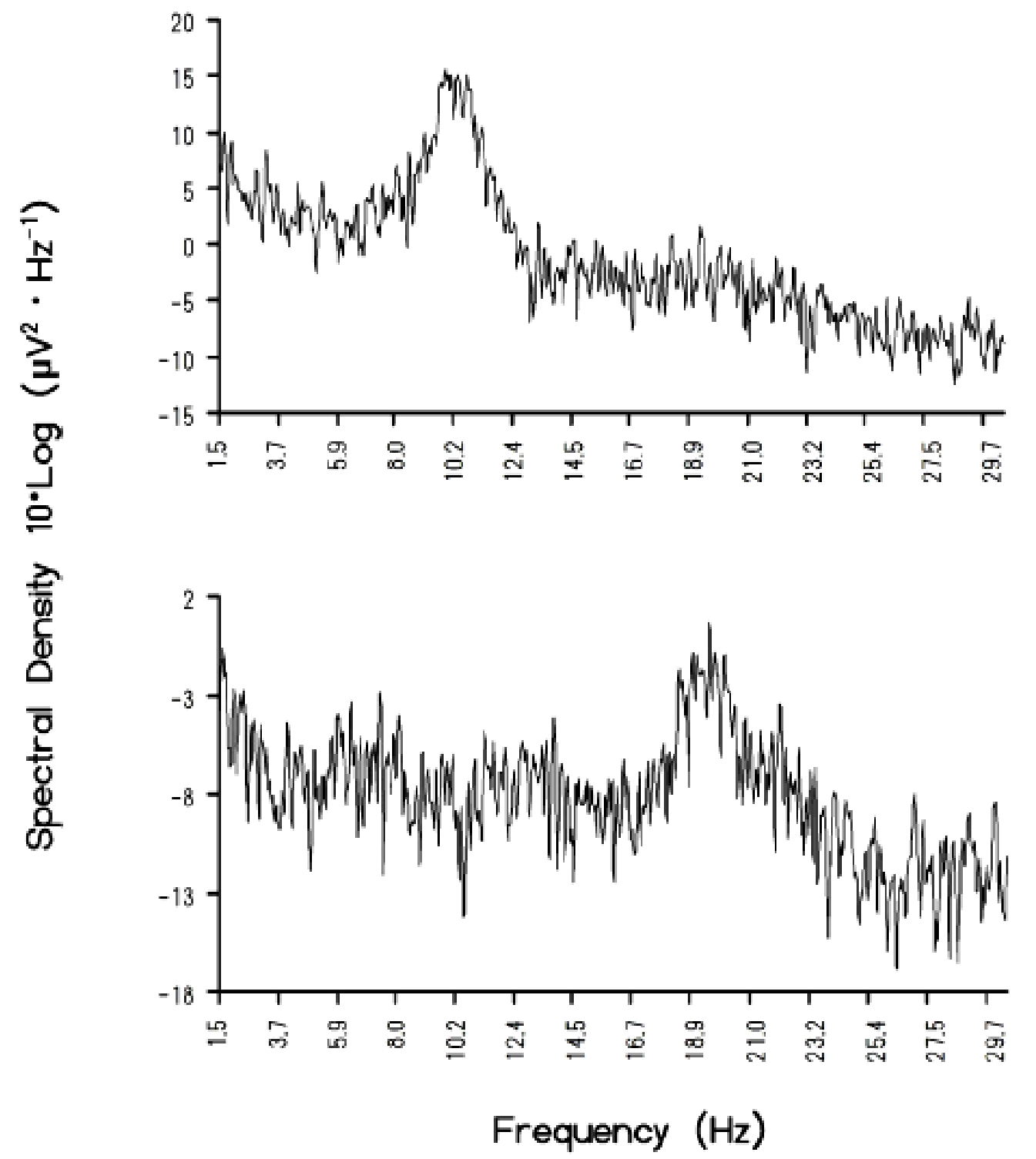

Figure 1. (Above)Sample power spectra of a classical quantitative EEG of an average person and (B) Schumann resonance profile associated with the same segment of quantitative electroencephalographic activity.

The primary source of the electromagnetic activity measured from the scalp emerges from the cerebral cortices because of the parallel arrangement of the dendrite-soma-axon orientations perpendicular to the surface for most of the approximately 20 billion neurons. The resulting steady state potential between the cerebral surface and a relative reference such as the lateral ventricle ranges between 10 and $20 \mathrm{mV}$. There is almost a linear correlation between ontogeny, the emergence of neuronal processes, and the magnitude of that potential.

Superimposed upon the steady potential are fluctuating voltages that define the electroencephalogram (EEG). Most of the discernable frequencies occur within the ELF (extremely low frequency) range or 1 and $100 \mathrm{~Hz}$. However, fast frequencies up to 300 to 400 $\mathrm{Hz}$, approaching the absolute refractory period of an axon, have been measured in epileptic 
brains [11]. An example of a traditional spectral density from an average adult human brain is shown in Figure 1.

The amplitudes of the scalp EEG range between 10 and $100 \mu \mathrm{V}$. In comparison corticographic discharges exhibit amplitudes between 0.5 and $1.5 \mathrm{mV}$ [12]. The most prominent dynamic pattern is the alpha rhythm ( 8 to $13 \mathrm{~Hz}$ ) which during wakefulness is more evident over the posterior cerebral space with amplitudes in most $(68 \%)$ of the population between 20 and $60 \mu \mathrm{V}$.

The average frequency increases from about $4 \mathrm{~Hz}$ in the 4 month old to $10 \mathrm{~Hz}$ by 10 years of age. The mean frequency in adults is $10.2 \pm 0.9 \mathrm{~Hz}$. Within the adult human alpha frequency slows between 0.3 and $1 \mathrm{~Hz}$ after ovulation. Amplitude asymmetry favors the right hemisphere in normal people [12].

Theta rhythms (4-8 $\mathrm{Hz}$ ) have been considered "intermediate waves" that are significantly involved with processes associated with infancy and childhood as well as drowsiness and specific stages of sleep. The power of theta activity is evident even within the third decade ( 25 to 30 years of age) when the EEG parameters asymptote. This inflection is sometimes considered an index of cerebral maturation.

A transient $6 \mathrm{~Hz}$ to $7 \mathrm{~Hz}$ rhythm, which disappears by adolescence, is common over the frontal midline and is enhanced by mental tasks related to memory function. Theta activity is the prominent frequency band within which most of the power within the hippocampus, the critical structure for consolidation of "memory", is displayed. The power or proportion of voltage within the theta range is associated with hypnogogic (dreamy or "apparitional" transitions) states particularly in late infancy and childhood.

For adults drowsiness is associated with the disappearance of the alpha rhythm and the emergence of low voltage 2 to $7 \mathrm{~Hz}$ activity intermixed with 15 to $25 \mathrm{~Hz}$ bursts. The hall mark of deep drowsiness is the appearance of vertex waves over the posterior portion of the supplementary motor area of the frontal lobe along the interhemispheric fissure. These transients are analogous to Q-bursts recorded from the Schumann cavity [3]. They are transient events of natural radio pulses with durations between 0.3 and $1.5 \mathrm{~s}$ detected globally due to exclusively powerful lightning discharges. The amplitude of Q-bursts, like vertex waves, can exceed the background by a factor of 10 and emerge as elevations in the Schumann harmonics in spectral analyses.

The theta-alpha range occurs within the band of the fundamental Schumann harmonic. The second harmonic, $14 \mathrm{~Hz}$, also has an equivalent within the EEG that emerges during light sleep (Stage 2). Sleep spindles or sigma waves are trains or envelopes of synchronous activity between 12.5 and $15.5 \mathrm{~Hz}$ with a peak around $14 \mathrm{~Hz}$. As alpha rhythms dominate the posterior region of the cerebrum, spindles emerge primarily over the frontal and frontal midline.

The other classical "frequency" bands associated with the human EEG are delta (1-4 $\mathrm{Hz}$ ), which are the highest amplitude time-variations associated with Stage IV sleep, beta (13$30 \mathrm{~Hz})$ and gamma $(30$ to $50 \mathrm{~Hz})$ patterns. The ranges are effectively arbitrary and related to EEG features associated with particular behaviors or power densities.

Large areas of the cerebral cortices demonstrate integrated spatial and temporal characteristics. Two that are relevant to Schumann interfaces involve the rostral-caudal "travelling waves" that are recursively (recreated) every 20 to $25 \mathrm{~ms}$ with an implicit frequency of about $40 \mathrm{~Hz}$ [13]. The bulk velocity is about $4.5 \mathrm{~m} \cdot \mathrm{s}^{-1}$ [14]. Assuming an average cerebral circumference of $0.6 \mathrm{~m}$ the resonance frequency is $7.5 \mathrm{~Hz}$, or within range of the fundamental harmonic of the Schumann Resonance. Llinas and Pare [15] also showed that 
there is a phase modulation of the $40 \mathrm{~Hz}$ recursive wave that was equivalent to $80 \mathrm{~Hz}$ or about $12.5 \mathrm{~ms}$.

The second major organization of human brain activity involves microstates which are defined by specific configurations of dipoles with isopotential lines over the entire cerebral cortices [16]. Most of the variance can be accommodated by four microstates whose patterns are similar across the human population and over ontogeny. According to convention these A, $\mathrm{B}, \mathrm{C}$ and $\mathrm{D}$ states are characterized by polarity from the right frontal to left occipital region, left frontal to right occipital region, equally distributed between the frontal and occipital region, and a dorsal central to caudal arrangement, respectively.

Together each state explains about three-quarters of the variance in EEG voltage variations. Each microstate's duration exits for between 80 and $120 \mathrm{~ms}$. These primarily four discrete and spatially-temporally bounded organizations could correspond to the "building blocks" for cognition and may be the basis for the unit "percept" of experience. This is equivalent to 8.3 to $12.5 \mathrm{~Hz}$, the inclusive band of the alpha range. Assuming these microstates are the "basic units" for thinking in a manner similar to the digital units (base pairs) for DNA [17] there would be $4^{10}$ or about $10^{6}$ possible different combinations or "serial patterns" per second.

The magnetic field component of human cerebral function is the same order of magnitude as the Schumann resonances. Although $\sim 10^{-12} \mathrm{~T}$ divided into $\sim 10^{-3} \mathrm{~V} \cdot \mathrm{m}^{-1}$ results in a velocity term that, with the appropriate coefficients, is the velocity of light (c), intrinsic magnetic properties of space can produce this value through spatial diffusion.

Magnetic diffusivity is:

$$
\eta \dot{\eta}=\left(\mu_{\mathrm{o}} \sigma\right)^{-1}
$$

where $\mu_{\mathrm{o}}$ is magnetic susceptibility $\left(4 \pi \cdot 10^{-7} \mathrm{~N} \cdot \mathrm{A}^{-2}\right)$ and $\sigma=$ electrical conductivity [18]. Assuming the average resistivity of $2 \Omega \cdot \mathrm{m}\left(\sigma \sim 0.5 \mathrm{~S} \cdot \mathrm{m}^{-1}\right)$ for extracellular fluid, and the average potential difference of $3 \mu \mathrm{V}$, the resulting estimate for the magnetic field strength is $\left(3 \cdot 10^{-6} \mathrm{~kg} \cdot \mathrm{m}^{2} \cdot \mathrm{A}^{-1} \cdot \mathrm{s}^{-3}\right)$ divided by $1.58 \cdot 10^{6} \mathrm{~m}^{2} \cdot \mathrm{s}^{-1}$ or $\sim 1.9 \cdot 10^{-12} \mathrm{~T}$ (about 2 picoTeslas, $\mathrm{pT}$ ).

This is well within the range based upon direct measurement from of action potentials from isolated frog sciatic nerves at distance of $1 \mathrm{~mm}$ [19].

The spatial domain is sufficiently large to have major influence upon the integrated cerebral field. This magnitude is within a factor of 10 of the transient magnetic oscillatory response Pantev et al [20] evoked in the human cerebrum (with a peak near $10 \mathrm{~Hz}$ and 30-40 $\mathrm{Hz}$ ) when their measured values for $20 \mathrm{fT} \cdot \mathrm{Hz}^{-1 / 2}$ are applied over the full frequency range of $100 \mathrm{~Hz}$.

Although these intensities appear small, experimental application of pT-intensity, spatial and temporally-patterned magnetic fields have been reported to produce discernable improvement in some clinical disorders, such as Tourette's and multiple sclerosis [21] as well as some electrical foci associated with partial complex seizures [22].

As shown by Booth et al [23] correlative responses associated with subjects' experiences that suggest more input from the right temporal lobe into the left temporal lobe (the sense of a presence) have been associated with geomagnetic changes in the order of $\mathrm{pT} \cdot \mathrm{s}^{-}$ ${ }^{1}$. The latter occurred when ambient geomagnetic changes displayed progressive increases at this rate over an approximately 15 min interval. 


\section{METHODOLOGY AND MATERIALS}

In order to compare directly the properties and interactions between human electroencephalographic activity and Schumann resonances, sensitive equipment is required. For the brain activity the standard 10-20 sensor array directly connect to a Mitsar 201 Amplifier System. The standard configuration is all sensors are referenced the ears, i.e., "monopolar" recording.

The induction coil employed to detect the Schumann frequencies was modeled after a designed outlined by Hans Michlmayr (http://www.vlf.it/inductor/inductor.htim). The length of the coil was designed to be $80 \mathrm{~cm}$ with copper wire wrapped around a $5 \mathrm{~cm}$ pipe. Approximately $15 \mathrm{~kg}$ of $0.3 \mathrm{~mm}$ (AWG 28) copper magnet wire were wound onto the PVC pipe with an electric power drill as suggested by Michlmayr. Our "Herbert" (after H. L. König) induction coil magnetometer is shown in Figure 2. The copper wire wrapping procedure is shown in Figure 3.

Assuming the weight associated with one turn of the pipe was $144 \mathrm{mg}$, division by the total weight indicates there were between 96,000 and 97,000 turns. The total resistance of the coil measured after winding was $3.97 \mathrm{k} \Omega$. The induction was 1000 Henrys once $182 \mathrm{~cm}$ insulated welding steel sheets were placed inside of the coil's frame. The finished coil was then housed in a larger PVC pipe for protection.

The preamplifier circuit was modeled after the ICS101 magnetometer designed by Renato Romero who operates an open-source website for the monitoring of VLF frequencies. The circuit can be found at http://www.vlf.it/romero3/ics101.html. For our preamplifier we omitted the $0-30 \mathrm{~Hz}$ low-pass filter. When the magnetometer was connected the pre-amplifier operated with a gain of $40 \mathrm{db}$.

The signal was sent to a USB soundcard, recorded and spectral analyzed automatically by SpectrumLab software designed by Wolfgang Buscher. The free source is located at: http://www.qsl.net/dl4yhf/spectra1.html. The software contains filters (set between 0 and 55 $\mathrm{Hz}$ ) and a $60 \mathrm{~Hz}$ notch filter that removed power-line frequencies before it was encoded into a Microsoft WAVE file with the sampling rate set at $204 \mathrm{~Hz}$. For calibration purposes as well as for real-time coherence measurements between human electroencephalographic activity and the Schumann resonance, the output from the preamplifier was directed to the ECG input of a Mitsar-201 amplifier.

With the Mitsar interface, we calculated the gain ratio of the signal after amplification through the preamplifier was $3.85 \cdot 10^{1}$. Using this gain ratio we calculated the approximate magnetic field intensity by integrating Faraday's Law of induction or

$$
\mathrm{V}=-\mathrm{n} \cdot\left[\boldsymbol{\Delta}(\mathrm{BA}) \Delta \mathrm{t}^{-1}\right]
$$

where $\mathrm{V}$ is the voltage generated, $\mathrm{n}$ is the number of turns of the coil, $\mathrm{A}$ is the area of the coil and $\Delta \mathrm{B} / \Delta \mathrm{t}$ is the changing magnetic field intensity in Teslas per second. Substituting the appropriate values for $\mathrm{n}(96,000$ turns $)$ and $\mathrm{A}\left(0.125 \mathrm{~m}^{2}\right)$ we obtained a constant by which $\mathrm{B}$ could be solved based upon the raw voltages from the Mistar interface at one of our station locations (Laurentian University soccer field).

When the signal's time series was spectral analyzed the approximate magnetic field intensity at $7.94 \mathrm{~Hz}$ was $1.21 \mathrm{pT}^{2} \cdot \mathrm{Hz}^{-1}$ or $3.09 \mathrm{pT}$ which is with range (Figure 4) reported by others $[3,24]$. 


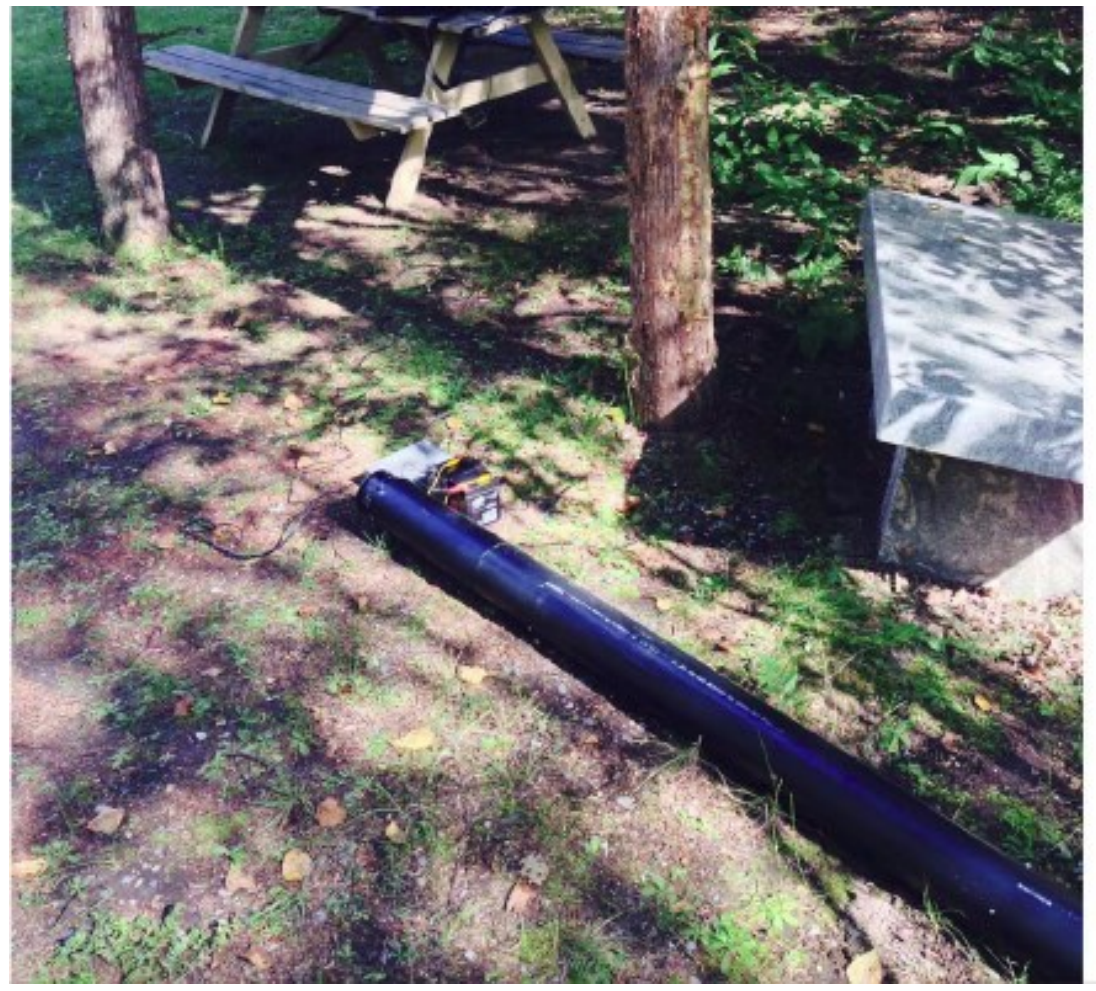

Figure 2. Cylinder containing the Schumann device "Herbert" by local Schumann resonances were measured.

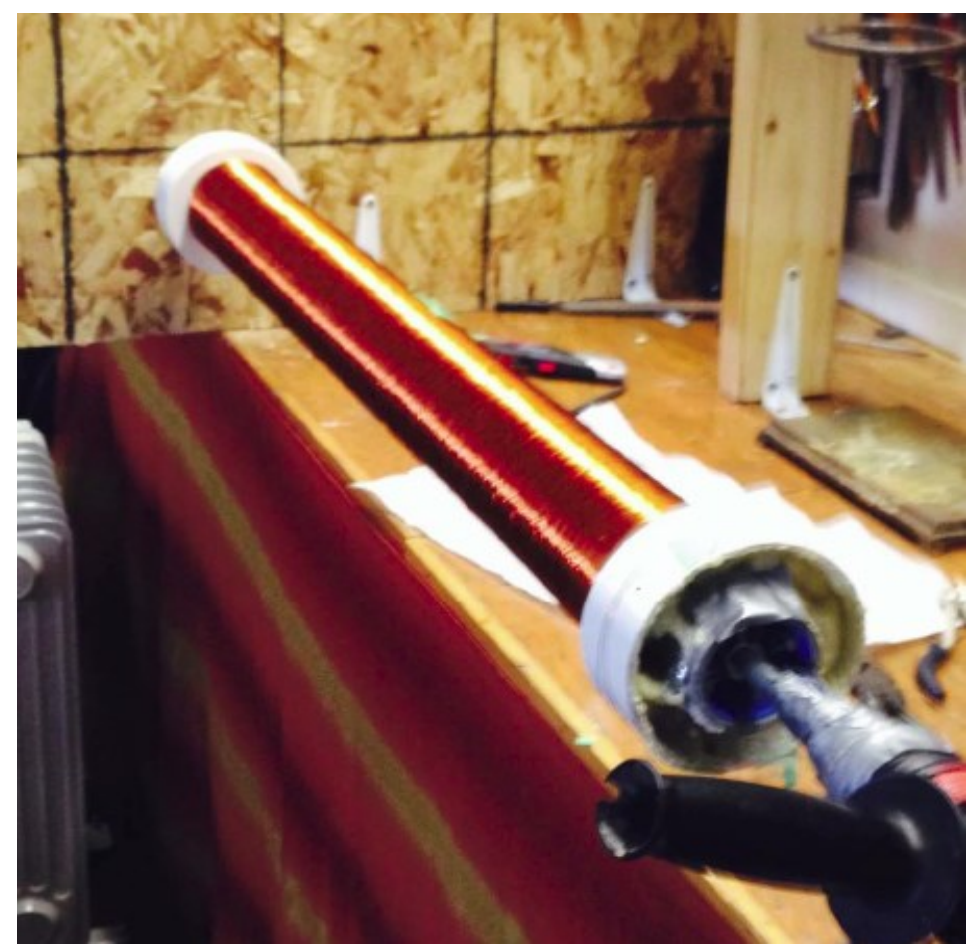

Figure 3. The Schumann ("Herbert") unit during stages of wrapping the copper wire. There were between 96,000 to 97,000 turns. 


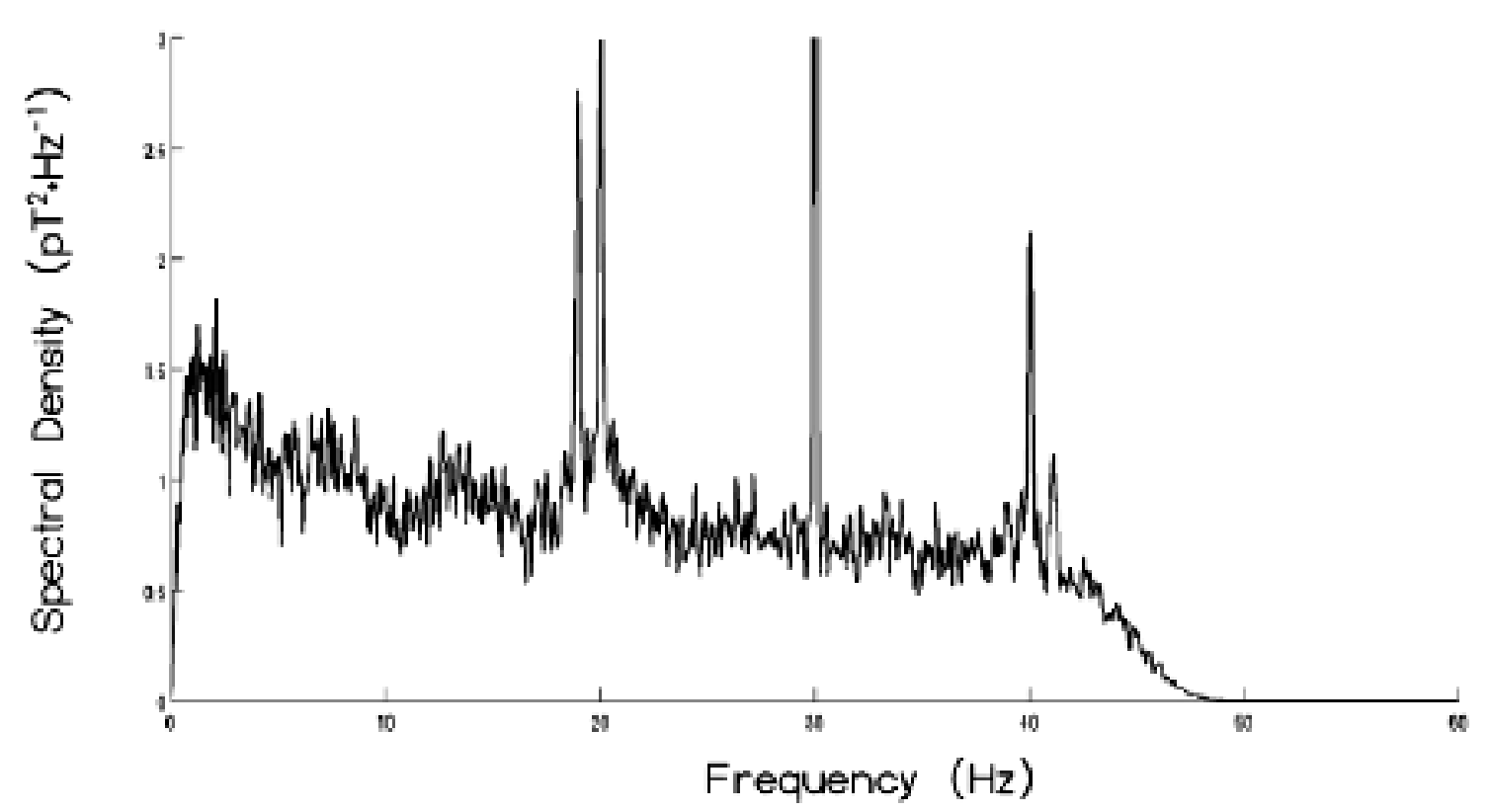

Figure 4. Calibration of the "Herbert" induction coil magnetometer demonstrating that the peak intensities at the harmonics were consistent with the measures of other stations. The mean peak values around $8 \mathrm{~Hz}$ is approximately $2 \mathrm{pT}$, within the range of other measurements.

\section{BASIC PATTERNS AND SPECTRAL FEATURES OF LOCAL SCHUMANN RESONANCES AND BRAIN REPRESENTATIONS}

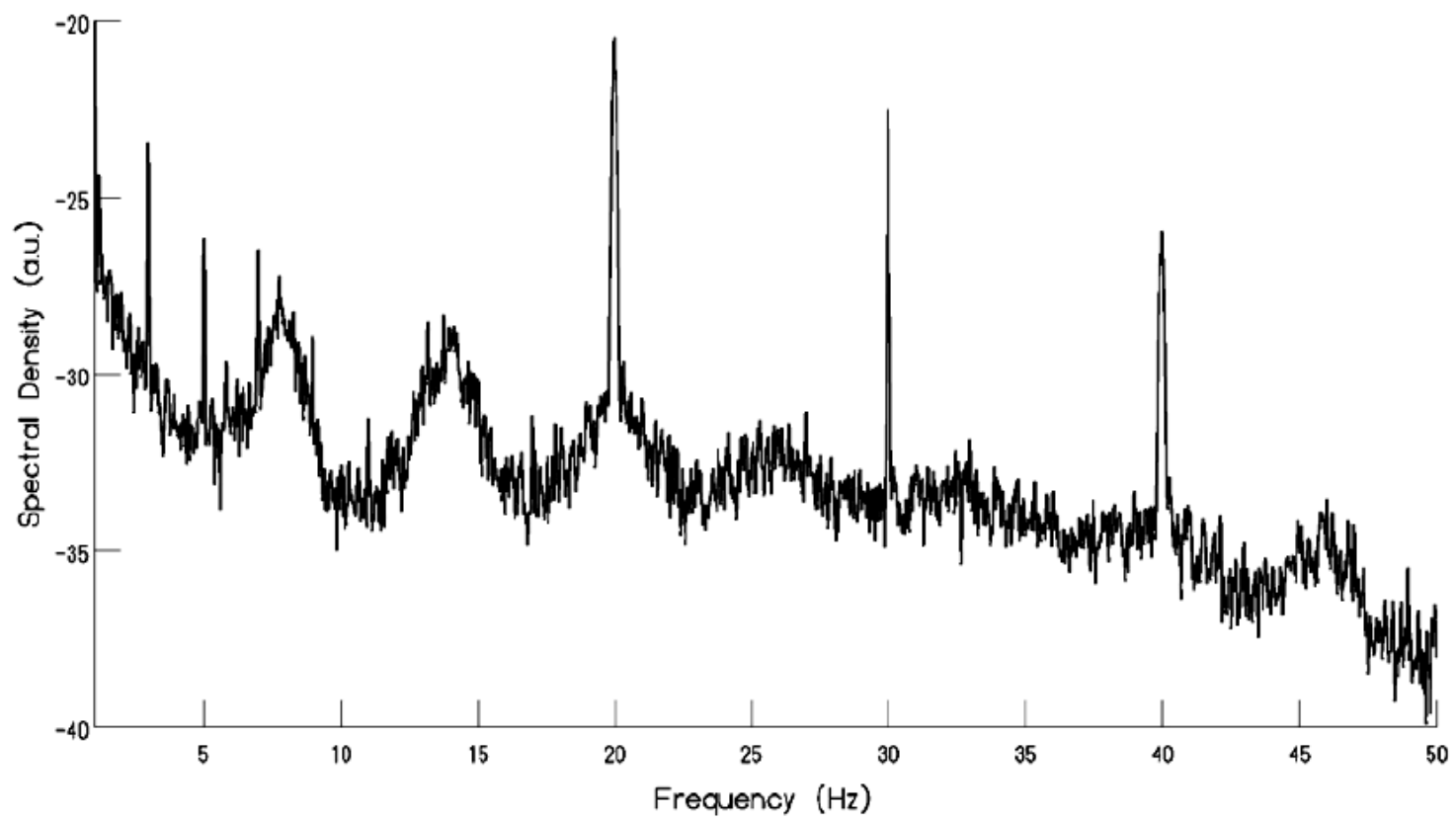

Figure 5. Log of spectral power density for Schumann resonances as a function of frequency from our local station. The relative harmonic peaks in arbitrary units (au) are veridical. Sharp spikes at some harmonic peaks may be actual. 
During fair weather conditions, the power density of the Schumann frequencies near our laboratory was similar to that reported from other researchers in the U.S.A., Italy, Russia and Japan.

An example of the spectral density of the magnetic field component in relative units and frequency is shown in Figure 5. The peaks are clearly evident at the fundamental and expand to the $7^{\text {th }}$ harmonic. However, by far the most conspicuous enhancement occurred for the first four harmonics.

The spectral analyses of the QEEG for our subjects have shown the presence of the Schumann resonances within cerebral cortices for potentials recorded between the left frontal and right occipital regions. In general the Schumann resonances are most prominent over the caudal portion of the cerebral measurements that include the occipito-parieto-temporal interface. As shown in Figure 6, the representations of the Schumann frequencies which are often recondite within the "noise" of the QEEG data become evident when filtering occurs. An example of a single participant's 'Schumann Resonance Signature' is shown in Figure 1.

The primary frequencies that appear within the caudal region were between 7-8 Hz, 13 $14 \mathrm{~Hz}$, and 19-20 Hz. Of the approximately 200 subjects measured under standardized conditions over the last three years only about $25 \%$ show the representation of the Schumann resonances within their EEG records.

Because all subjects (records) were treated similarly the likelihood of artifact for this representation of the Schumann resonances from sampling, filtering, or instrumental anomalies is minimal.

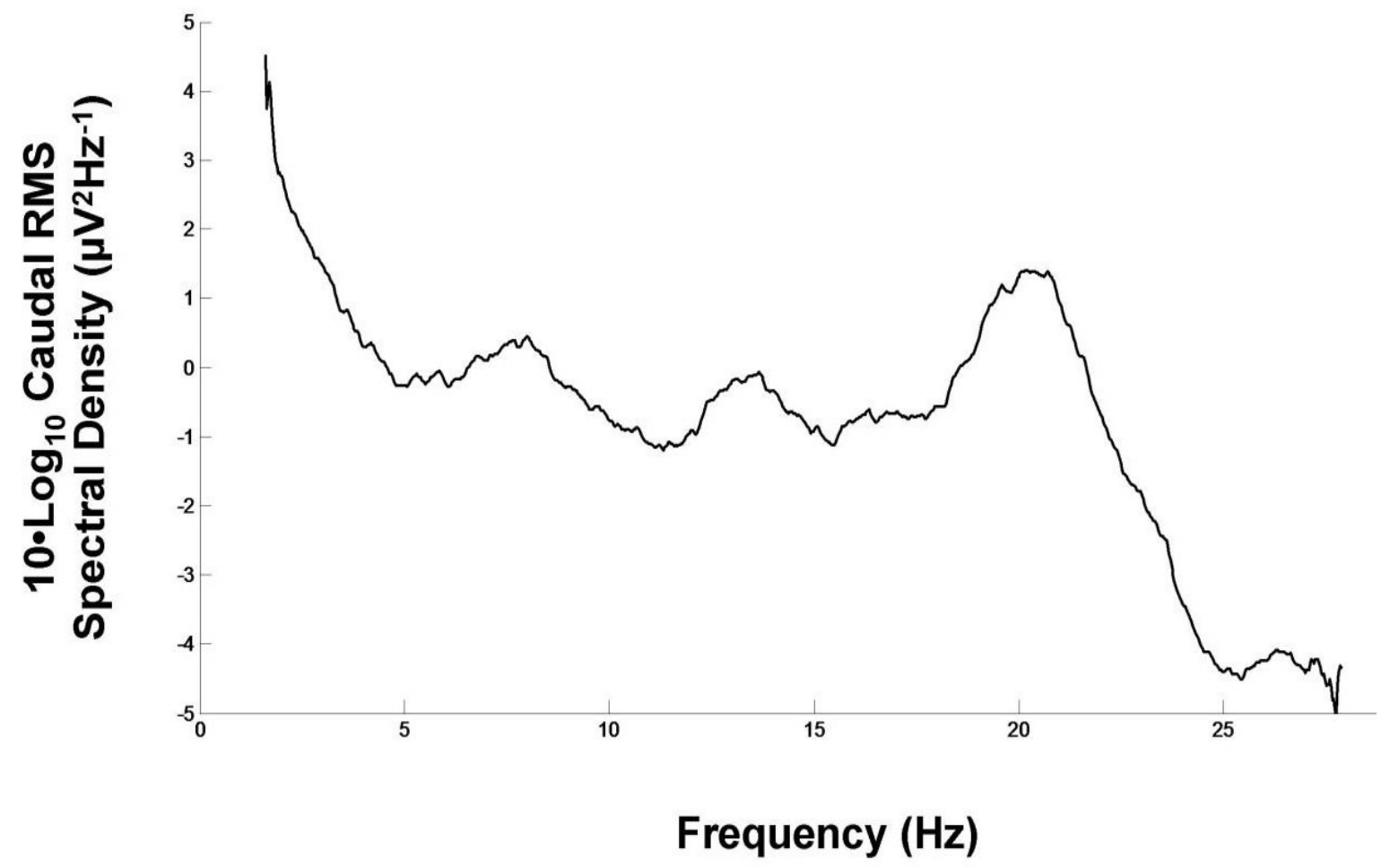

Figure 6. Spectral density over the caudal region (temporal-T5 T6, occipital O1 O2 and parietal, P3, $\mathrm{P} 4, \mathrm{Pz}$ ) of 34 subjects whose brain activity was measured while sitting in a quiet Faraday chamber. 


\section{TEMPORAL INTEGRATION OF SCHUMANN FREQUENCIES MAY BE SIMILAR TO MUSICAL FUGUES}

The potential information contained within the Schumann frequencies has not been frequently or systematically pursued. Even a cursory inspection of actual raw data from direct Schumann resonances suggests that if it were a musical score, it may display the features of a "fugue". A fugue is a form of music in which a subject melody (usually only about 5 to 10 notes) is repeated but in combination with different harmonics over time.

To discern if there are "higher order" arrangements of Schumann patterns, the a 300 second signal recorded through the Schumann coil was directed to the USB soundcard and imported into MATLAB where the signal was decomposed into harmonic signals by filtering the original signal within frequency bands characteristic of Schumann eigenfrequences with bandwidths of $+/-$. $5 \mathrm{~Hz}$ around center frequencies of 7.8, 13.8, 20.2, 33, 39 and $45 \mathrm{~Hz}$. The most conspicuous result was the detection of spindle-like occurrences primarily within the 14 $\mathrm{Hz}$ range, but also apparent in other frequency bands, which was apparent from preliminary testing with data collection through the Mitsar amplifier. This is the same range (13-15 Hz) as Stage II spindles measured over the human brain (particularly over the frontal regions) that has defined the interface between waking and light sleep [11]. The similarity between the Schumann spindles and the human cerebral spindles that define Stage 2 sleep was discovered and articulated many times by one of Schumann's students H. L. König [4]. An example is shown in Figure 7 which shows spindle-like activity among various harmonics of the Schumann resonance.

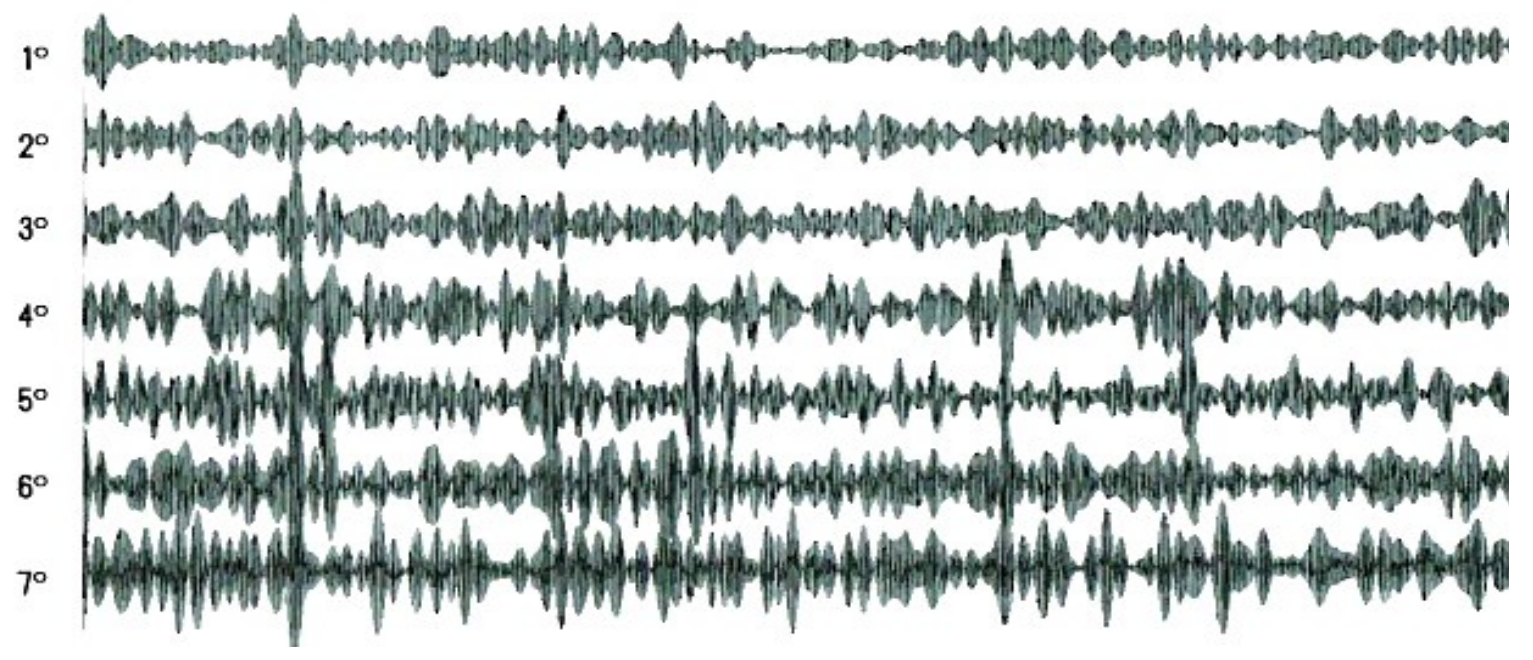

Figure 7. Pattern of spindle-like activity recorded locally by a Schumann unit after filtration within each of the Schumann resonance modes. Each line represents a harmonic of the Schumann resonance starting with the fundamental $7.8 \mathrm{~Hz}$ (top line) and ending with the $7^{\text {th }}$ harmonic $45 \mathrm{~Hz}$ (bottom line).

Next a Hilbert Transform was applied to discern the envelopes ("spindle amplitudes") for each of the signals. The results are shown in Figure 8. The resultant signal, from a recording that was approximately 2 hours in duration, was then re-filtered between 0 and $2 \mathrm{~Hz}$ to focus primarily on the changes within the envelope over time. These data were entered into coherence analysis to discern if any "fugal" coupling occurred between each resonance. 


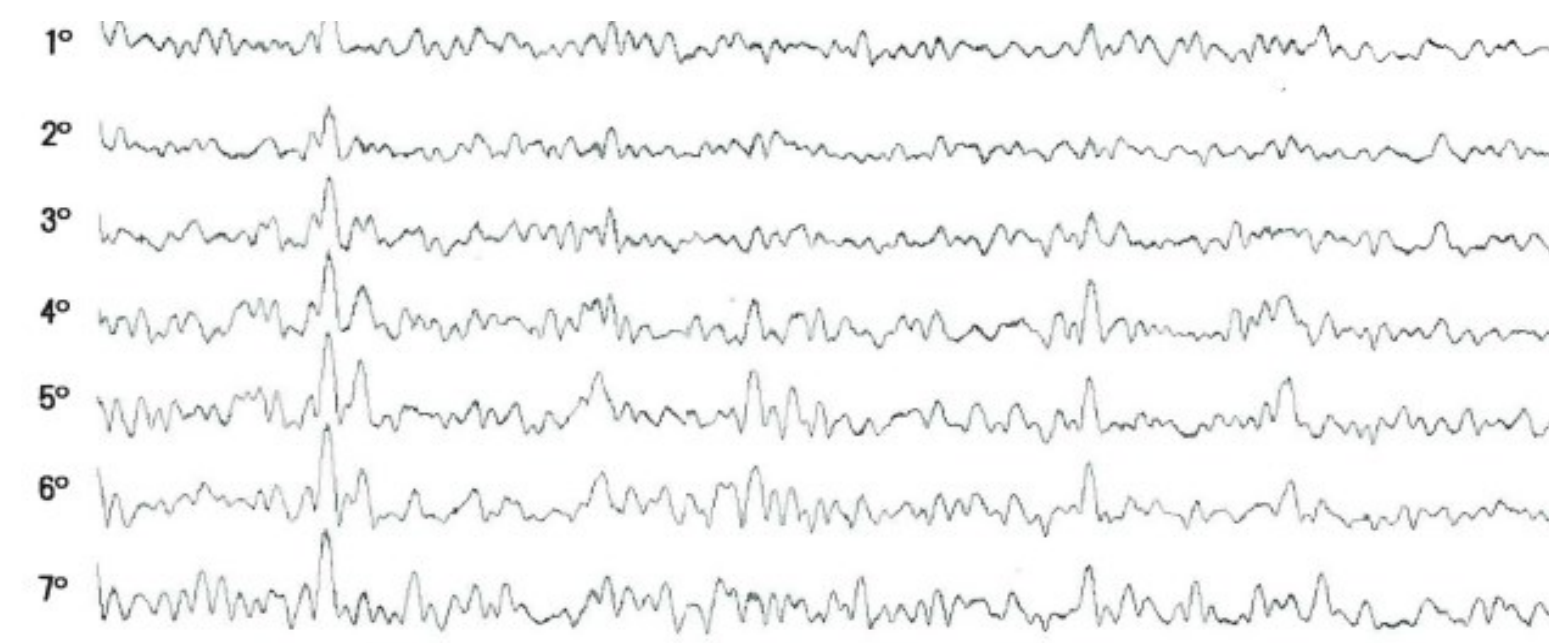

Figure 8. Frequency pattern within each envelope or spindle from Figure 6 after Hilbert transformation.

The results, in Figure 9, reveal a clear coupling between harmonics of the Schumann resonance, in this instance between 7 and $45 \mathrm{~Hz}$. This couplet of frequencies, 7 and $45 \mathrm{~Hz}$, is within the range of the coupling of activity found in the most fundamental activity of the hippocampus [25]. This brain structure is essential for the formation of the representation of particularly the spatial features of experiences that define semantic, episodic and autobiographical memory. The "pacemaker" cells for the two frequencies displayed by the hippocampus have been attributed to the midline structure between the two lateral ventricles, the septum.

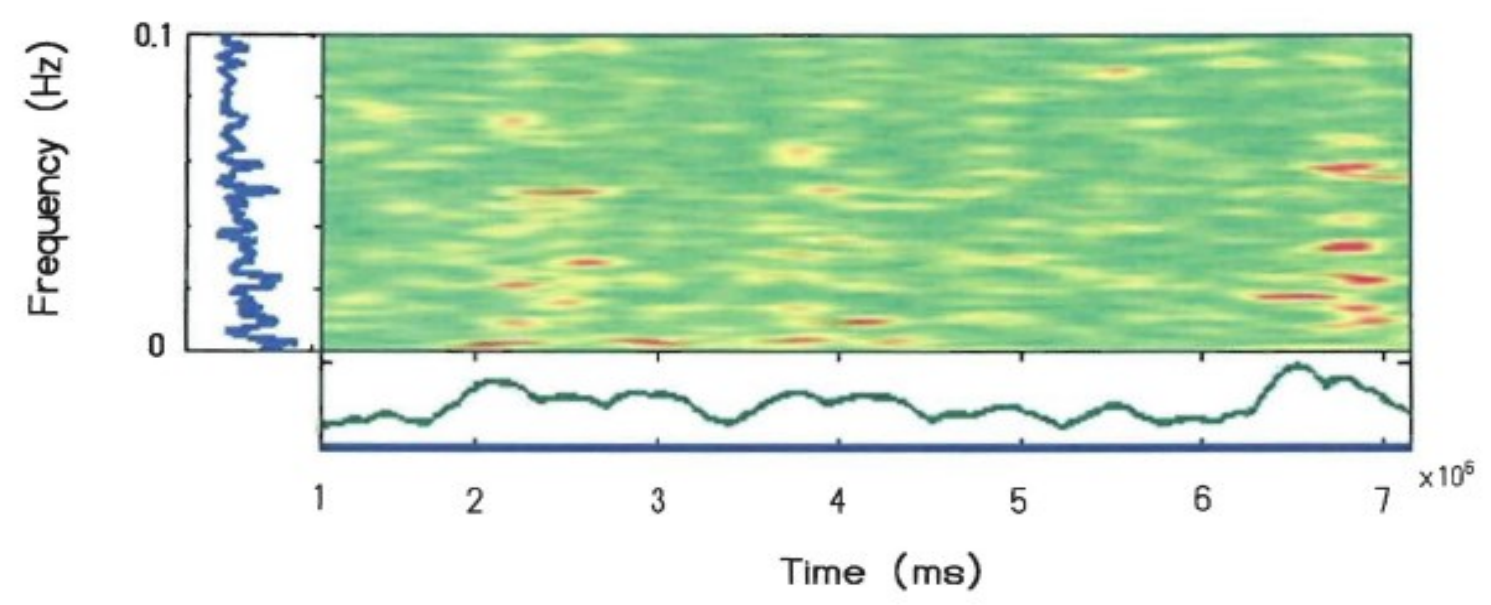

Figure 9. Demonstration of a type of fugal coupling between the various harmonics of the Schumann values. This example depicts coupling specifically between the first and seventh Schumann harmonics. Red and yellow areas indicate cross-frequency coherence intervals.

The intrinsic high amplitude population voltage of $\sim 7 \mathrm{~Hz}$ (theta range) within this mesiobasal structure within the ventral portion of the temporal lobe effectively defines the dynamic properties of the structure. Superimposed upon this fundamental frequency are much 
smaller amplitudes or "ripples" that range between 40 to $45 \mathrm{~Hz}$. Several researchers [26] have proposed that the $45 \mathrm{~Hz}$ ripples superimposed upon the theta activity within the hippocampus allows the interaction between "memory" and "consciousness".

The structure of the hippocampal formation, the technical definition of the hippocampus proper and the dentate gyrus [27], is important because they are effectively a small spherical condenser wrapped and partially interdigitated by a larger spherical condenser. This $\mathrm{C}$-shaped structure is similar to a toroid with a gap which allows a discrete leakage of magnetic flux. According to classic electromagnetic theory geometries exhibiting this configuration are strongly affected by polarization or phase vector [28].

The numbers of neurons within the human hippocampal formation are in the order of 1 to $3 \cdot 10^{7}$. Burke and Persinger [29] have shown quantitatively at least, that this number of neurons contains the potential to converge with parameters of fundamental constants such as Planck's mass and the hydrogen line.

The emergence of a similar temporal coupling between $7 \mathrm{~Hz}$ and $45 \mathrm{~Hz}$ patterns in the Schumann and cerebral domains suggests that occasionally resonance might occur between the two sources. Because the magnetic field strengths of the first Schumann harmonic and the human brain are in the order of $2 \mathrm{pT}$ shared energy occurs. The magnetic energy within the human cerebral volume estimated by:

$$
\mathrm{E}=\mathrm{B}^{2} \cdot(2 \mu)^{-1} \mathrm{~m}^{3}
$$

where $B$ is the strength of the field, $\mu$ is the magnetic permeability and $\mathrm{m}^{3}$ is volume, results in $2.1 \cdot 10^{-21} \mathrm{~J}$.

The energy lost when 1 bit of information is removed into entropy (or gained if accessed from this source) according to Landauer is $\ln 2 \mathrm{kT}$, where $\mathrm{k}$ is the Boltzmann constant $\left(1.38 \cdot 10^{-23} \mathrm{~J} \cdot \mathrm{T}^{-1}\right)$ and $\mathrm{T}$ is the temperature in degrees $\mathrm{K}$ for brain temperature is $\sim 4.3 \cdot 10^{-21} \mathrm{~J}$. This convergence suggests that the energy shared by both the magnetic field strength of the first harmonic of the Schumann resonance and the human cerebrum are equivalent to the threshold where bits of information are dissipated into or acquired from entropy. At this threshold intercalation of energy sources may not be restricted necessarily by conventional limits of impedance, masking by thermal agitation, the "weak" strength, or distance.

If the volume of both left and right hemispheric hippocampal formations $(6 \mathrm{cc})$ were substituted for the total cerebral volume the energy equivalence would be $\sim 9.6 \mathrm{~J} \cdot 10^{-24} \mathrm{~J}$. However the power $\left(\mathrm{J} \cdot \mathrm{s}^{-1}\right)$ required to approach the Landauer threshold would require a frequency equivalent to the duration between 1 and $3 \mathrm{~ms}$, the approximate duration of an action potential. This value is the shortest interval for inter-hemispheric communication and is the duration for the expansions of electrons and protons by one Planck's length [29]. Thus from a simplistic resonance approach, the loss or gain of bits of information between the Schumann sources and the human brain, would require this weak intensity magnetic field and the fluctuating range of perturbations that are routinely generated by the human brain.

Although 1 bit per second could be considered minimal, within $24 \mathrm{hrs}$ the information would be within the tens of kilobytes. Within a $15 \mathrm{~min}$ period, the time required for the growth of a new dendritic spine in the hippocampus and cerebral cortices and the spatial representation of a "memory", the potential shared information would be in the order of 1 kbit. This would be sufficient to affect the occurrence or non-occurrence of information during the ephemeral period (about 15 minutes) of memory consolidation which can be quickly "erased" by excessive electrical stimulation. When the research by Li, Poo and Dan 
[31] is considered that experimentally demonstrated that the activity of only one neuron can affect the state of the entire cerebral cortices, such small values are not trivial.

Additional analyses indicated in Figure 9 indicate that incidence rate at which the couplings occurred between the 7 and $45 \mathrm{~Hz}$ Schumann sources. The vertical axis displays the frequency at which the couplings occur while the horizontal axis reveals the numbers of times they occur. The value was about 2 to 2.5 times per $1000 \mathrm{~s}$ or approximately once every 7 seconds. Hence even if the estimates for direct addition or removal of information is diluted by a factor or about 10 , there would be sufficient intercalation to affect neuronal processes at the synaptic level.

Figure 9 suggests that these complex analyses of direct measurements of local Schumann features reveal temporal clusters. If the envelopes of spindles are considered analogous to "subject melodies" then the frequencies "harmonize" with each other over time. The second and third order derivatives or "harmonies" could have the potential to interact with similar changes within the human brain volume. In other words the mode of interaction between the ionosphere-earth cavity and the cerebral cortices would me mediated through the fugal patterns rather than the single frequency resonances.

\section{CORRELATION BETWEEN REAL SCHUMANN AND CEREBRAL COHERENCE VALUES}

One of the most common comments concerning the "impossibility" of the potential interaction between or representations of Schumann resonances within human brain activity as defined by QEEG is the very weak magnitudes of the vertical electric field (about 0.1 to 1 $\left.\mathrm{mV} \cdot \mathrm{m}^{-2}\right)$ and the horizontal magnetic field $(\sim 2 \mathrm{pT})$ for both sources. Yet most physical perspectives one smaller volume (the human cerebrum) immersed within a much larger volume (the earth-ionospheric shell) should share quantitative values for primary parameters. This would be expected for organic systems that have been organized, evolved and maintained this system since abiogenesis [32].

Empirical support for this expected interaction should be apparent considering the sensitivity of modern QEEG and Schumann technology. There are several processes that could facilitate interactions. If they occurred and equilibrium $(B+E=k)$ occurred between the Schumann (E) and brain (B) sources, negative correlations would be expected between the magnetic field and electric field strengths for the harmonics for the Schumann modes and corresponding power or distribution of "energy" within specific frequency intervals or "bands" within the quantitative electroencephalographic activity for the human brain.

If an immersion model were valid it might be tested by the effect of the more expansive source (the transglobal Schumann field strengths) upon the coherence of the more localized source (the human brain). Coherence can be defined as the proportion of congruence between power spectra of one source with that of another. It is typically expressed as a correlation coefficient or its squared value which is an indicator of shared temporal variance. Hence coherence values range from 0 to 1 .

Coherence is functionally a juxtaposition of two or more or "identical" complex temporal patterns that are usually displaced in time with respect to each other. One common description is "phase-shifted". If two sources with "identical" complex oscillations are phase shifted completely by 90 degrees, the average would be a form of cancellation that would emerge as "random" noise. However if the two patterns from the two sources were shifted to 
overlap precisely within each other at the same "time", then the coherence would be maximum and the average strength of the coherence would be maximum.

Coherence from shifting phases requires, usually, minimal energy because the larger proportion of energy that constructs the complex patterns is not changed. What is changed is the energy of the dynamic process that produces the rate of change which will determine when the patterns will occur in real time within each locus as well as the transposition of axes. In this situation each locus is each cerebral hemisphere. This is often reflected as a first or second derivative and is implicitly associated with symmetry of a process moving in a circle.

The immersion model predicts that as the intensity of the primary field (the Schumann sources) increases, the intercalation between disparate but coupled asymmetrical processes in a more or less equal volume should increase. For the human brain the two volumes refer to the left and right hemisphere. On average the volumes of the cortices of the two hemispheres (right larger) differ by about $10 \mathrm{cc}$. The effect should be most evident between the maximum widths of two hemi-ellipsoids which would occur approximately at the level of the temporal lobes.

The dynamic asymmetry of the human brain has been attributed to the approximately 10 $\%$ greater white-to-grey matter ratios and blood flow in the right hemisphere which is slightly larger and heavier than the left. The structural component for most interactions between the two hemispheres, primarily the corpus callosum and anterior commissure, is composed of numbers of axons that are less than $1 \%$ of the numbers of neurons within the cerebral cortices of either hemisphere. Hence there should be specific latencies for quantities of information to be transmitted between the two loci.

Effectively the human cerebrum is two boundaries or loci within which processes associated with more than $90 \%$ of the neurons in each locus remain and do not interact directly with the homologous area in the other hemisphere. The differences in morphological structure between the two cerebral hemispheres have been underestimated. As shown by Van Essen and Drury [10], only four of the shapes and lengths of the approximately 176 sulci and gyri that comprise both human cerebral hemispheres are very similar. If structure dictates function than the two hemispheres should have qualitatively different functions whose integration or interface is limited by the fidelity and accuracy of interhemispheric communication.

"Awareness" or "consciousness" in the classic "awake" sense involves a dynamic dominance of more left hemispheric activity information. During this state the temporal patterns (information) from right hemisphere must be represented within left hemispheric space. The representation, because of the function of the left hemisphere, would be primarily the images associated with verbal or symbolic labels.

The congruence or accuracy between stimuli preferentially affecting the right hemisphere and the experience should be determined not only by the pattern of reinforcement history of the individual (in large part determined by culture) but by the proportion of coherence between the activities of the two hemispheres within millisecond time. Direct measurements indicate that the shortest interhemispheric time for action potential propagation from one area to another is in the order of 2 to $3 \mathrm{~ms}$ [33].

For the present experiment, the coherence strength between the left (T3) and right (T4) temporal lobes from routine QEEG measurements from human volunteers were calculated for those days in which the electric field strength data were also available. The electric field waveform data for this study were accessed through the Northern California Earthquake Data Center (NCEDC). The instrument (BQ2) was an electric field dipole situated at Jasper Ridge Station (JRSC) in Stanford California USA, which recorded the electric field intensity $(\mathrm{V} / \mathrm{m})$ 
40 times per second. Only data between August 2009 and August 2010 was used for this study because the Schumann resonance 8 and $14 \mathrm{~Hz}$ peaks were readily visible after spectral analyses for these data.

As can be seen in Figure 10, there were moderate strength correlations between the proportion of coherence strength between the left (T3) and right (T4) temporal lobes around $11.6 \mathrm{~Hz}$ and the average Schumann electric field strength (in $\mathrm{mV} \cdot \mathrm{m}^{-2}$ ) for its first harmonic ( $\mathrm{r}$ $=0.43$, rho $=0.53)$ and second harmonic $(\mathrm{r}=0.45$, rho $=0.51)$. This pattern is conspicuous even by visual inspection. The fact that the brain activity was measured in Sudbury, Ontario, Canada and the electric field measurements of the Schumann data were recorded in Italy supports the concept of the global nature of the effect.

Within the $95 \%$ confidence band for the linear regression, the minimum to maximum change in interhemispheric coherence involved an increase of $0.2 \mathrm{mV} \cdot \mathrm{m}^{-1}$ in the global field strength. The slope was 0.32 and 0.41 respectively indicating that for every $0.1 \mathrm{mV} \cdot \mathrm{m}^{-1}$ increase in the Schumann E field, coherence increased by 0.32 and 0.41 within the potential range of 0 to 1 .

If a similar field strength was induced within the human cerebral cortices, the potential difference would be $2 \cdot 10^{-4} \mathrm{~V} \cdot \mathrm{m}^{-1}$ over the cerebral length of $10^{-1} \mathrm{~m}$ or $2 \cdot 10^{-5} \mathrm{~V}$. Assuming the classic resistivity of extracellular fluid within the brain of $2 \Omega \cdot \mathrm{m}$, the current would be $10^{-5}$ $\mathrm{A} \cdot \mathrm{m}^{-1}$ or, over the averaged length of the cerebrum, $10^{-6} \mathrm{~A}$.

This current value when divided by $1.6 \cdot 10^{-19}(\mathrm{~A} \cdot \mathrm{s}) \cdot \mathrm{q}^{-1}$ (unit charge), indicates that the change would be equivalent to $0.6 \cdot 10^{13} \mathrm{q} \cdot \mathrm{s}^{-1}$. Because approximately $10^{6}$ unit charges are associated with the membrane potential [34], $\sim 0.6 \cdot 10^{7}$ neurons could be involved. The estimated numbers of soma whose axons compose the corpus callosum and that intercalate the functions of the left and right hemispheres are about $2 \cdot 10^{8}$.

On the other hand if only the fibers within the dorsal hippocampal commissure were considered [35], approximately 0.5 to $0.7 \cdot 10^{7}$ neurons would be involved. This is remarkably similar to the numbers of neurons within the hippocampal formation. Because the dynamic changes within the parahippocampal gyrus are strongly affected by the hippocampal input and this gyrus can influence the entire cortical manifold, this small number of neurons could affect hemispheric coherence.

Thus, a first order estimate indicates that the proportion of neurons affected by a Schumann-matched voltage shift would be $\sim 1 \%$. This small percentage is within the range required to produce the slight shift in discharge frequencies that would allow the population convergence of activity between the left and right hemispheres. That our results were largest for the temporal lobes (or a region strongly coupled to it) indicates that his region could be a preferential locus for the influence from the ambient Schumann intensities or a region strongly coupled to it.

The strongest correlation between the increased electric field of the first two Schumann harmonics and the $11.6 \mathrm{~Hz}$ bin for coherence between the left and right temporal lobes could suggest the emergence of a "beat" frequency from the first two harmonics within brain space.

If the first and second harmonic of the Schumann sources were $7.8 \mathrm{~Hz}$ and $14.1 \mathrm{~Hz}$, the beat would be approximately $11 \mathrm{~Hz}$, assuming equal biases for the intensities. The "11.6" $\mathrm{Hz}$ bin is within the normal variation of the classic peak alpha power in human EEG profiles. The width of most power curves before attenuation into background variations is between $\sim 7$ to $14 \mathrm{~Hz}$. 


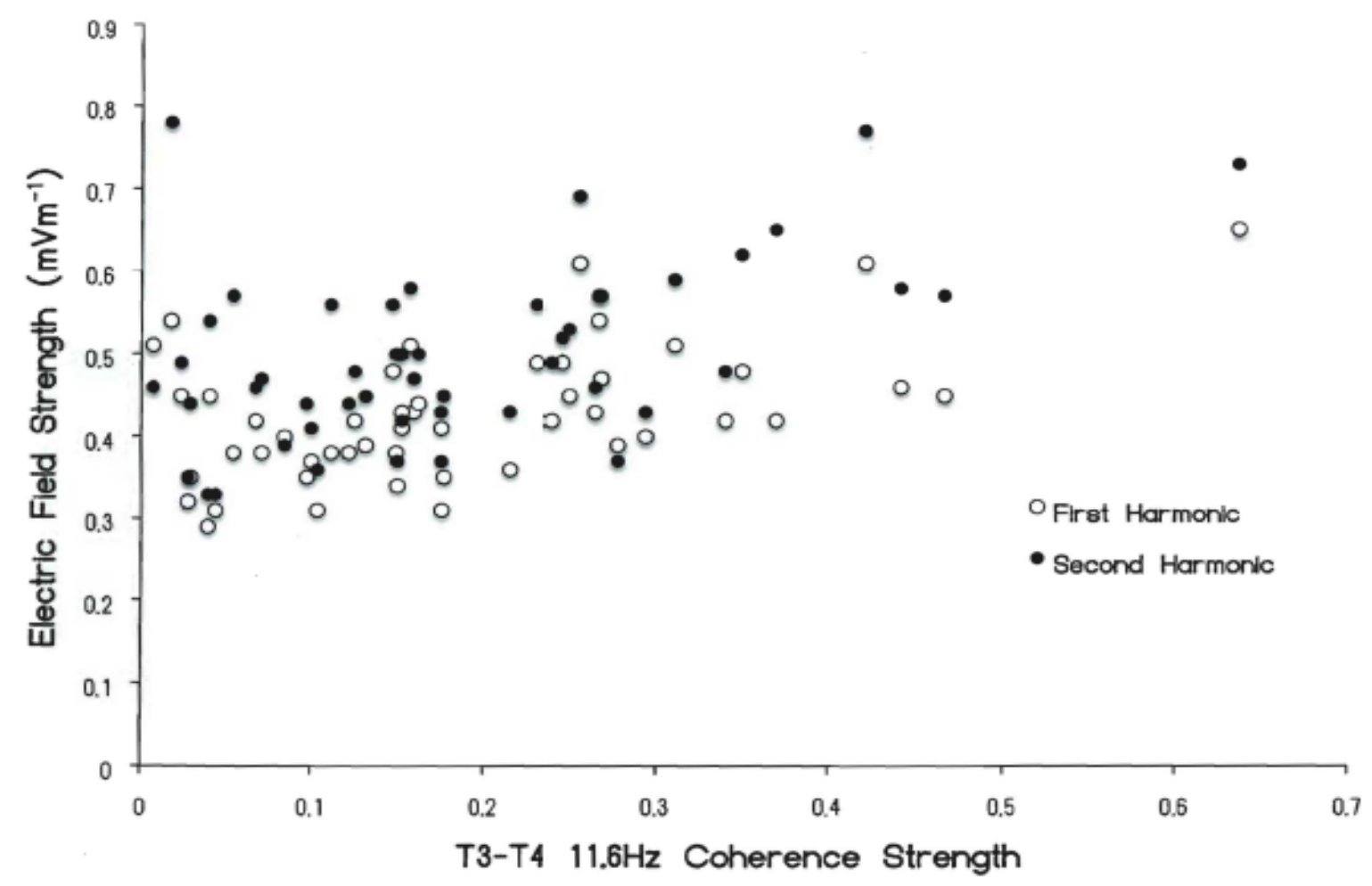

Figure 10. Correlation between the coherence strength between the left (T3) and right (T4) temporal lobes for subjects whose QEEGs were measured in Sudbury, Ontario and the electric field strength of the first two Schumann harmonics in California.

\section{THE PARAHIPPOCAMPAL REGION AS A LOCUS OF INTERACTION}

The maximum correlation between Schumann electric field shifts and the coherence between the left and right temporal lobes compared to other regions should reflect a structural or dynamic difference compared to other regions of the brain. The ventral portions of the temporal lobe are unique in that interhemispheric intercalation is primarily determined through the anterior commissure rather than the corpus callosum only. The hippocampal formation, the gateway to the representation of experiences (memory), is connected interhemispherically through the dorsal hippocampal commissure. It occupies the anterior one third of the splenium of the caudal corpus callosum.

In addition the middle and particularly the inferior temporal gyri are strongly influenced by the parahippocampal gyrus. This area of the human brain may be essential for the transduction and transformation of Schumann frequency changes for several reasons. First, neurons within the two upper layers of the entorhinal cortices that form a significant portion of the parahippocampal region (and the uncus) display an intrinsic (subthreshold) oscillation with a peak around $8 \mathrm{~Hz}$. The prominent sub-threshold oscillations ranged between $5.5 \mathrm{~Hz}$ and $14.5 \mathrm{~Hz}$ [36] which would include the first and second Schumann harmonics.

This region is unique structurally. Layer II, or the layer of star cells (stratum stellare of Stephan) exhibits large, dark staining stellar-shaped neurons with spine-covered dendrites radiating into all three planes of space. The neurons in Layer II are clustered in "islands" or "bands" which define the characteristics of the entorhinal cortices. These protrusions on the cortical surface ("verrucae gyri hippocampi") are visible macroscopically. Fibers originating 
from these cells terminate in the dentate gyrus before the information is propagated to the hippocampus proper [37].

The mean amplitude of these subthreshold oscillations averaged $2.6 \mathrm{mV}$. This periodic increment of potential difference upon a unit charge is equivalent to $4.2 \cdot 10^{-21} \mathrm{~J}$ of energy which is within the Landauer limit for the loss or gain of one bit of information into or from entropy. The access to energy contained within the "random" processes that defines entropy may reflect a component of the Zero Point Vacuum Fluctuations [38].

These phenomena, especially when Casimir forces are considered, are interconnected with the transformation of virtual particles into real particles and could alter the limit for interaction between and information of the "weak" electric and magnetic fields shared by the Schumann and brain sources. As stated by Bordag et al [39] the creation of particles from a vacuum requires energy transference from the external field to the virtual particles or vacuum oscillations. However the boundary conditions must be dynamic or dependent upon time.

Secondly, neurons within the parahippocampal gyrus, particularly the upper two layers of the entorhinal cortices, are the major pathway to and from the hippocampal formation. Third, through a cascade of corticocortical projections the entire manifold of the cerebral cortices converges within the second and third layers of the entorhinal cortices. This allows all of the activity of the cerebral cortices to be affected by and to affect the entorhinal area. A specific coupling or resonance between the Schumann fields and this portion of the human brain could affect the entire cerebral cortical manifold for protracted durations. These durations are juxtaposed and could certainly exceed the time required to form new synapses and patterns of neuronal connections that define and determine "memory". From some perspectives a person is defined by his or her memories.

The parahippocampal region preferentially relays more spatial information, an essential component for most memories. It contains dominating reciprocal connections with the prefrontal cortices, especially the dorsolateral aspect. Consequently changes within the parahippocampal region could alter the major cerebral region associated with reasoning, selfmonitoring and decision-making. The second major input, from the cingulate, would add the component of emotion of the type commonly associated with bonding to another human being.

Fourth, the parahippocampal region is an intermodality integrator where "line codes" from different sensory modalities such as vision and hearing are transformed to equivalent and potentially interactive frequencies. E. R. John [40] had shown experimentally several decades ago that learning through different modalities was transferable in a single trial if the frequency patterns of the pulses from different modalities were identical. Interactions of input from different modalities information could be synthesized into experiences.

The intensities of the Schumann resonance E-field $30 \mathrm{~min}$ before to $30 \mathrm{~min}$ after the time of 16-s resting eyes-closed QEEG records for 49 subjects measured from August 2009 to August 2010 were used for this analysis. The e-field data waveform data was accessed from the NCDEC as previously described. Derivations of the absolute difference in voltages between the right and left parahippocampal region from 1 to $35 \mathrm{~Hz}$ and the amplitude of the mean activity between 7.8 to $8 \mathrm{~Hz}$ were correlated.

Preliminary analyses indicated that there was a conspicuous increase in correlation strength between the first Schumann harmonic and $9-10 \mathrm{~Hz}$ activity within the parahippocampal region. The strength of the Pearson and Spearman correlations were -.50 and -.49, respectively. In other words as shown in Figure 11, as the intensity of the fundamental 7.8 to $8 \mathrm{~Hz}$ Schumann frequency increased the absolute discrepancy between the right and left parahippocampal regions decreased. 
This would be consistent with an increased coherence between the two hemispheres as observed between the left and right temporal lobes.

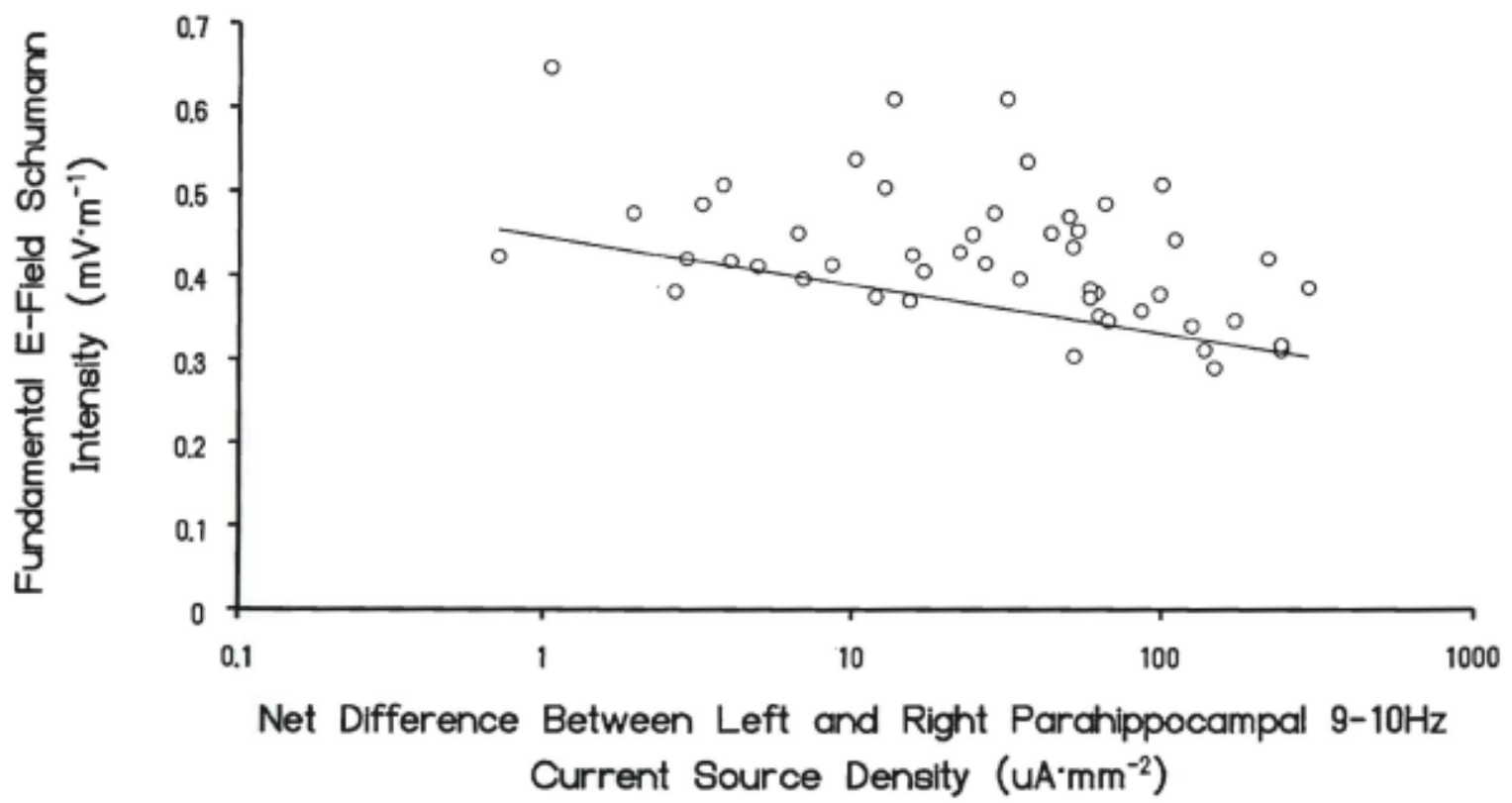

Figure 11. Correlation between the intensity of the contemporary fundamental electric field of the Schumann resonance in California and the net difference in current density as measured by sLORETA for the 9 to $10 \mathrm{~Hz}$ band between the left and right parahippocampal regions for 49 subjects whose QEEGs were measured between 2009 and 2010.

It is also evident that an absolute shift in current density ratios between the right and left parahippocampal region of more than 100 units $\left(1\right.$ to $\left.100 \mu \mathrm{A} \cdot \mathrm{mm}^{-2}\right)$ was associated with a range change of only about $0.2 \mathrm{mV}$ in the electric field component of the fundamental Schumann frequency. Approximately $10 \mu \mathrm{A}$ within a cross-sectional area occupied by the parahippocampal region convergences with the activity of the numbers of neurons likely to be involved with intercalation of left and right temporal lobes. This occurred with an increase of only $0.1 \mathrm{mV} \cdot \mathrm{m}^{-1}$ of the Schumann electric field.

If the amplitude of the fundamental Schumann frequency was in the lower range, the current density would be 100 times larger. This would indicate the right hemisphere was more active than the left. Even when general metabolic activity or peak-to-peak voltages of alpha rhythms are considered the "default" condition is for more energetic utilization or potential difference within the right hemisphere compared to the left.

If the interaction between the Schumann and cerebral cortical voltages were in the right hemisphere, then the resonance would facilitate a reduction in current density. The diminished current density in the right parahippocampal region might be dissipated extracerebrally, that is, in the direction of the ionospheric-earth resonance cavity.

We have not correlated the relationship between simultaneous electric or magnetic field components of the Schumann resonance and cerebral power or coherence during REM (rapid eye movement) or dreaming conditions. These periods occur once every approximately 90 to $120 \mathrm{~min}$ for about 5 to 10 minutes during the first episode to 10 to 20 minutes towards the termination of the sleeping period. During REM there is a marked increased in cerebral blood 
flow and general cerebral metabolic activity, including the protein synthesis associated with memory consolidation, as well as cardiac arrhythmia.

An equilibrium model would predict that during this period there should be an increase in current density within the right parahippocampal region as the Schumann amplitudes decrease. That would suggest the direction of this metaphoric "Poynting vector" would be towards the intracerebral locus. The direction of the equilibrium would be from the ubiquitous Schumann sources into the right hemisphere. Thus over the diurnal period there would be an oscillation or vectorial property with respect to the direction of the brain-Schumann interaction.

\section{EXTENDED CORRELATIONS}

Considering the caudal position of the splenium and the dorsal hippocampal commissure, one would expect a more likely representation of Schumann frequencies within the caudal portions of the human cerebrum. This predominance has been supported directly by our QEEG measurements. To discern the relationship in greater detail a data set consisting of spectral densities $(\mathrm{N}=18)$ from the caudal root-mean-square of the posterior channels (T5, P3, Pz, T6, O1, O2) as well as the spectral densities for the electric field measured in Italy were combined and compared. The electric field data was provided courtesy of Mr. Renato Romero who continuously monitors very-low frequency electric field perturbations with a Marconi-T antenna.

Canonical correlations between the various frequencies from this cerebral composite and the spectral densities of the E-field data from the Schumann frequencies indicated that $14.65 \mathrm{~Hz}$ or the presence of the second Schumann harmonic within brain activity was a strong predictor. Both parametric and non-parametric correlations showed consistent negative correlations. The effect sizes, the square of the correlation coefficient, when graphed as a function of frequency displayed peaks that were very similar to Schumann resonances with the strongest effects for the first three harmonics.

Figure 12 shows this association. The effect size was about 0.4 , indicating that $40 \%$ of the variance was shared between the route-mean-square of the posterior brain activity and the range of the Schumann electric field. The overlap is particularly prevalent for the second and third harmonic. The second harmonic has been shown mathematically to be a possible interface between gravitational waves and the earth's ionospheric-earth cavity [41].

The increased coherence between left and right temporal lobes by an increase of only $0.1 \mathrm{mV} \cdot \mathrm{m}^{-1}$ may be critical for cosmological interface. A comparable change in potential over the cerebral cortices, assuming a length of $10^{-1} \mathrm{~m}$, is $1 \cdot 10^{-5} \mathrm{~V}$. The effect on a unit charge of $1.6 \cdot 10^{-19} \mathrm{~A} \cdot \mathrm{s}$ would be $1.6 \cdot 10^{-24} \mathrm{~J}$. The quantum frequency, obtained by dividing by Planck's constant $\left(6.626 \cdot 10^{-34} \mathrm{~J} \cdot \mathrm{s}\right)$ is $2.4 \cdot 10^{9} \mathrm{~Hz}$ which is very proximal to the neutral hydrogen line that permeates the universe.

When the correlations between cerebral spectral density and the Schumann E fields were lagged and lead, the associations were still statistically significant within a three day window. Additional lag/lead analyses indicated that the relationship was no longer statistically significant for two weeks. This suggests that the temporal interval for direct coupling between the cerebral and terrestrial sources could be limited. Interesting 10 days was the first peak in power density for spectral analyses of Schumann intensities that were not related to solar effects according to Nicolaenko and Hayakawa [3]. 


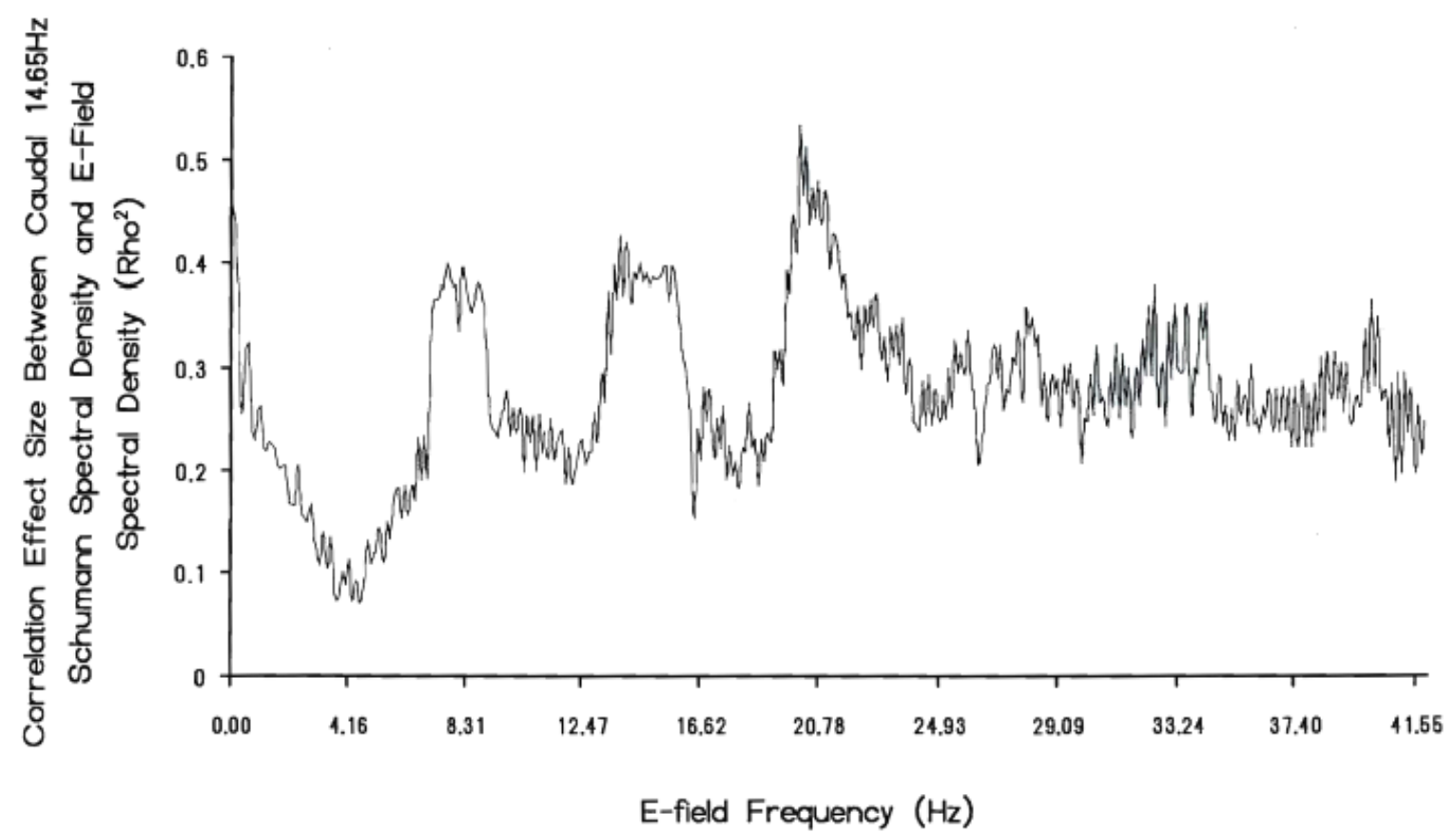

Figure 12. The effect size (correlation coefficient squared) for the association between the caudal 14.7 Hz QEEG activity in Sudbury and Schuman E field spectral density in Italy and the frequency band of the E field. Note the strongest correlation for the first three harmonics of the Schumann resonance.

If "gravity" waves share a similar source of variance to Zero Point Vacuum Potentials [37], then the "coincidental" similarities of the threshold Landauer value for information dissipation into entropy or its emergence from it and the amplitude fluctuations of the $\sim 8 \mathrm{~Hz}$ subthreshold variations could reflect one intercept between human brain activity, the Schumann frequencies and "gravitational" phenomena.

Although gravitational phenomena are often considered too weak to be of significance for biological matter, there is quantitative evidence that the energies are within the range of those relevant to cell membranes [42] and interactions with cerebral-electromagnetic interactions [43]. The alternative explanation for gravitational phenomena and dark matter developed by Borowski [44] suggests there are relationships yet to be discerned.

Applications of the calculations by Ahmed et al [45] to bulk cerebral cortical velocities of $4.5 \mathrm{~m} \cdot \mathrm{s}^{-1}$ indicated that the effects of gravity as inferred by weightlessness upon the human EEG would be about $\sim 3$ to 4 parts per 10 million. When divided by the common frequency in the universe, $1.42 \mathrm{GHz}$, the emergent duration would be 20 and $30 \mathrm{~ms}$ or the recursive cerebral waves associated with consciousness. This interesting solution suggests that the subtle effects of gravity or its intrinsic variations [46] could interface with the phasemodulations of cerebral activity.

Phase-modulation has often been considered the most effective means to propagate the most information over great distances. One solution is:

$$
\mathrm{t} /\left(\mathrm{v}^{2} \cdot \mathrm{c}^{-2}\right)
$$

where, $\mathrm{t}$ is the reference time, $\mathrm{v}$ is the velocity and $\mathrm{c}$ is the velocity of light. Because the median frequency of the electromagnetic fields associated with lightning, the sources of the 
Schumann resonance, is between $10 \mathrm{kHz}$ and $100 \mathrm{kHz}$, the $\Delta \mathrm{c} \cdot \mathrm{c}^{-1}$ is 0.05 according to Tu et al [47]. This indicates that the means of the phase shifts for every second would be $\sim(0.9897)^{-1}$ or in the order of 15 to $20 \mathrm{~ms}$. This value is congruent with the phase comparisons of approximately 10 to $20 \mathrm{~ms}$ associated with the $\sim 40 \mathrm{~Hz}$ integrated waves that spread over the entire cerebral cortical mantle. Quantitatively, the conditions are set for resonance interactions and exchange of information between the cerebrum, the ionospheric-earth cavity, gravitational waves, and potentially astronomical sources.

\section{IMPLICATIONS OF SCHUMANN AMPLITUDE FACILITATION OF INTERHEMISPHERIC COHERENCE IN THE HUMAN BRAIN.}

Our calculations indicate that only a $0.1 \mathrm{mV}$ per $\mathrm{m}$ increase in the electric field of the global Schumann fundamental frequency is sufficient to increase the coherence from the lower one-third to the upper one-third of the potential range between the left and right temporal lobes of the human brain. If this can be generalized then the involvement of $0.6 \cdot 10^{7}$ neurons with implicit or active shifts in potential of $\sim 10^{-20} \mathrm{~J}$ per s associated with a given action potential would be $0.6 \cdot 10^{-13} \mathrm{~J}$.

Over the cross-sectional area of the cerebral cortices $\left(\sim 10^{-2} \mathrm{~m}^{2}\right)$ the $0.6 \cdot 10^{-13} \mathrm{~J} \cdot \mathrm{s}^{-1}$ would be equivalent to a flux density of $\sim 0.6 \cdot 10^{-11} \mathrm{~W} \cdot \mathrm{m}^{-2}$. This value is within range of fluctuation of measurement error for the increases in photons that emerge from the right hemisphere when people sitting in hyper-dark chambers engage in imagination of white light compared to more mundane thoughts [48]. This convergence could suggest that the processes involved with this light emission from the human brain is coupled to the degree of coherence and dynamic intercalation between the two hemispheres rather than more direct metabolic activity of cerebral cortical activity.

In the sample population of approximately 200 brains that were measured by QEEG within more or less the same conditions (in a quiet, Faraday room, darkened and subject instructed to close eyes and relax), only 34 subjects exhibited strong Schumann resonances intensities in the spectral densities of their 10-20 sensor array. Such individual differences would be expected if this phenomenon were like any other natural phenomenon. If the data are representative of the human population than about $25 \%$ of the population would exhibit electroencephalographic power densities that reflect the Schumann harmonics.

The most conspicuous appearance of the Schumann resonances within the brain power densities occurred for the caudal regions of the cerebrum. This would be consistent with a particular sensitivity of this region. We suggest that the source of this sensitivity may be to the influence from the parahippocampal region because the actual locus for the dorsal hippocampal commissure is located within the anterior one-third of the splenium of the corpus callosum. The splenium is the primary source of interhemispheric fibers that intercalate visual, auditory-visual, and visual-spatial information between the left and right hemispheres.

If the primary means by which the effects of the Schumann-related intensities and information are mediated between the "dreaming" and "unconscious" right hemisphere and the left hemisphere (associated with awareness and consciousness) is the dorsal hippocampal commissure within the anterior one-third of the corpus callosum, then "memory" would be one of the primary areas of influence for the Schumann-cerebrum interaction. It is relevant that electrical lability or (limbic) epileptic events involving this pathway produce a single, powerful effect: amnesia [35]. 
A less extreme condition where mass depolarization would not occur but rather the normal flow of information between the hippocampal structures would only be modulated by Schumann-sourced electromagnetic fields could modify the memory consolidation. The retrieval and experience of "a memory" that is different from a fantasy, a dream, or an actual representation of experience involve the integrity and integrative activity of the right prefrontal region. The modified "consolidations of experiences" could be experienced as actual memories.

\section{DIRECT PROOF OF PRINCIPLE OF REAL-TIME COHERENCE BETWEEN BRAIN ACTIVITY AND SCHUMANN RESONANCES}

Although the moderate correlations between the averaged values per day for the measures of $\mathrm{mV} \cdot \mathrm{m}^{-1}$ (vertical) from the Schumann measurements at a distance and QEEG activity completed in our laboratory strongly supports effects from distal sources, a real timecoupled demonstration was ultimately required. For this measurement a volunteer lay in supine position approximately $1 \mathrm{~m}$ away from and parallel with the Schumann (Herbert) system shown in Figure 2. The subject wore the 10-20 sensor cap (Electro-Cap International; $19 \mathrm{AgCl}$ sensors). Thirty (30) minutes of simultaneous data were collected.

The input from the sensory (EEG) cap and the input from the induction coil magnetometer were directed to the Mitsar 201 amplifier (input range $\pm 500 \mu \mathrm{V}, 16$ bit analogue-digital conversion) system so the raw voltage fluctuations in real time could be visualized. An example of the results is shown in Figure 13.

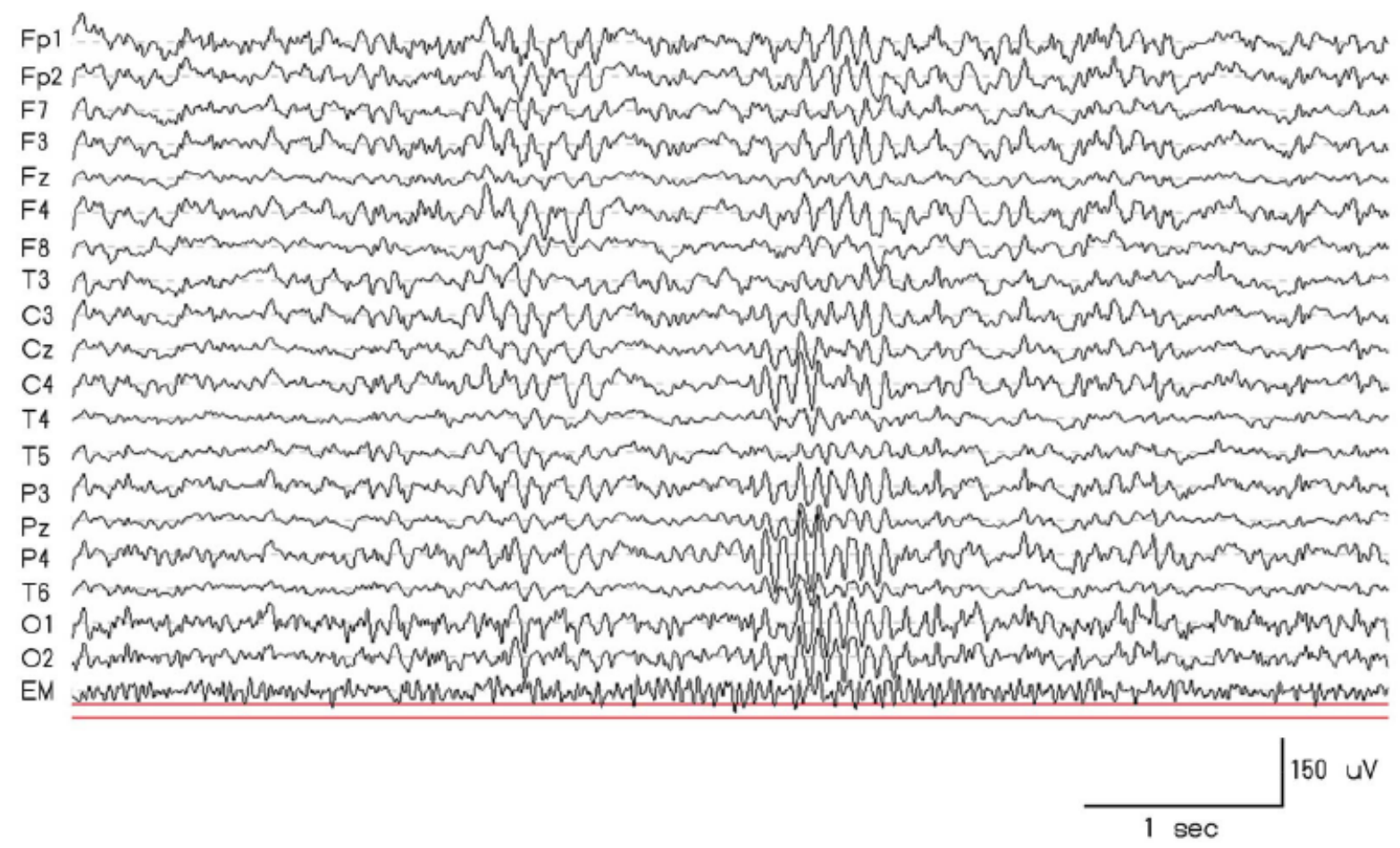

Figure 13. Sample (about $5 \mathrm{~s}$ ) electroencephalographic voltage pattern from the different sensor sites over the skull (indicated by traditional identifiers) and the voltage fluctuations associated with the simultaneous recording of the Schumann resonance. 
A sixty (60)-second segment of EEG/EM data for which the Schumann resonance was readily apparent within the EM signal during initial spectral screening within WinEEG was exported. Once imported into MATLAB the EEG data were filtered between 1.5 and $40 \mathrm{~Hz}$ and caudal root mean-squared signals were obtained by averaging the square of posterior sensors (T5, P3, Pz, P4, O1, O2) in accordance with our usual methods. The caudal RMS as well as the signals from the magnetometer were then consolidated and z-scored. These data were then entered into Delorme and Makeig's [49] EEGLab for the computation of channel cross coherence.

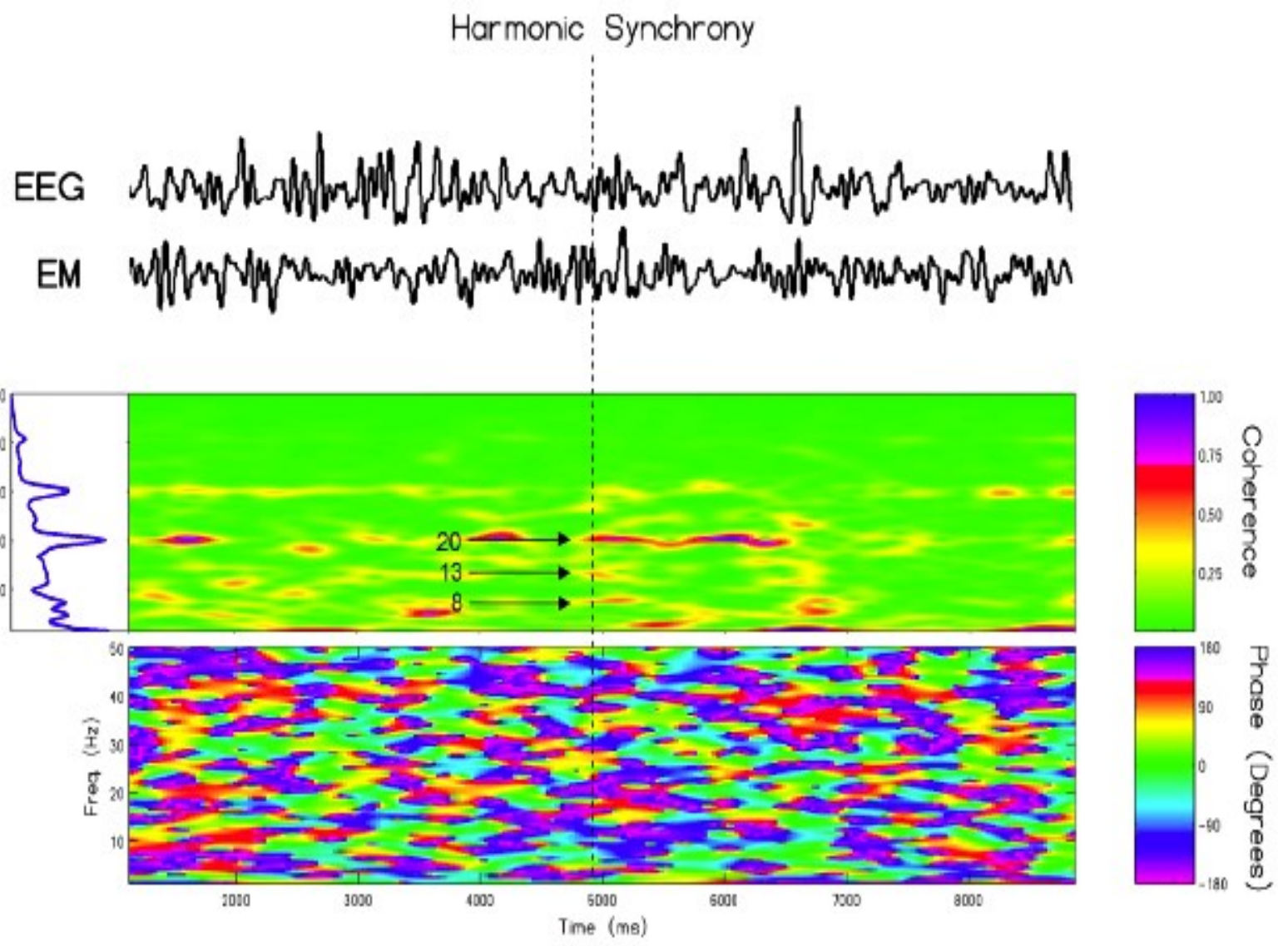

\section{$7.8 \mathrm{~Hz}$ Phase Shift: $41.9 \mathrm{~ms}$}

Figure 14. Sample record (about $10 \mathrm{~s}$ ) of the composite EEG from the caudal portions of the subject's cerebrum (EEG) and the Schumann resonance (EM) measured $1 \mathrm{~m}$ away. The horizontal axis is real time in ms while the vertical axis is frequency. The first, second and third harmonic of the Schumann resonance measured with the magnetometer were coherent with the 8,13 , and $20 \mathrm{~Hz}$ activity occurring within the integrated caudal root-mean-square signal. The yellow indicates weak $(0.30$ coherence while the red indicates stronger $(0.5$ to 0.7$)$ coherence. In the first panel (below the raw data) the coherence durations in the indicated frequencies occurred primarily as brief periods between $0.3 \mathrm{~s}$ to about $2 \mathrm{~s}$. The phase shift is noted in the lower panel. The phase shift for this event at $7.8 \mathrm{~Hz}$ with cerebral activity was equivalent to about $41.9 \mathrm{~ms}$. "Harmonic synchrony" or simultaneous coherence across two or more harmonics (possibly 4 or 5 with different magnitudes of coherence) is indicated by the vertical dotted line. 
The results of the coherence analyses are shown in Figure 14. "Harmonic synchrony" coherence between two or more frequencies at the same time involved four and perhaps 5 bands (if weaker coherence values are included). This synchrony is indicated by the vertical dotted line. The analyses revealed moderately strong coherence $(0.7)$ between the 8,13 and $20 \mathrm{~Hz}$ Schumann frequencies measured from the magnetometer and the power within the person's electroencephalographic activity at 8,13 , and $20 \mathrm{~Hz}$ respectively. The durations of the coherence were transient but consistent and extended between $0.3 \mathrm{~s}$ to about $2 \mathrm{~s}$ (red). The weaker coherences (yellow) were more protracted. Less frequent episodes of very brief strong coherence was evident in the $30 \mathrm{~Hz}$ range.

The phase shift (in degrees) of the coherence is shown in the multicolored bottom panel for this "Harmonic" event. The green indicates no phase shift, that is, a coupling between the $7.8 \mathrm{~Hz}$ Schumann fundamental and the various spectral power bands of the EEG. Qualitatively the periods of no phase shift suggested as irregular vertical patterns across most frequencies are interspersed with more irregular distributions over scattered frequencies at the $\pm 180^{\circ}$ shift. The mean phase shift for this subject was about $42 \mathrm{~ms}$ or $-118^{\circ}$ which indicated that, for example, the $7.8 \mathrm{~Hz}$ activity occurring within the earth-ionosphere lead the $7.8 \mathrm{~Hz}$ activity occurring in the caudal regions of the cerebrum.

To our knowledge this is the first demonstration of a functional and time-coupled relationship between the areas of the human brain associated with the representation of Schumann resonances and the actual real-time occurrence of these resonances. The moderate strength coherence value was similar to what was found for the brain activity of our larger population and Schuman E field values from Italy and California, based upon daily values. The present measurement involved effectively continuous $2 \mathrm{~ms}$ to $2 \mathrm{~ms}$ ( $500 \mathrm{~Hz}$ sampling for both the Schumann and brain activity) sequences and revealed similar strength concordance.

The fact that the periods of coherence between Schumann resonance and brain activity within frequencies that matched the Schumann harmonics were brief, ranging from about 0.25 $\mathrm{s}$ to about $2 \mathrm{~s}$ and irregular temporally is more typical of nature phenomena than of artifact. If they were related to a shared artifact the coherence would be have been continuous which it was not.

Although perhaps spurious we think it is interesting that the range of the strength of the coherence between the Schumann eigenfrequencies and the harmonics within the cerebral cortical activity of this subject would be consistent with the standard deviations of the magnetic component for the $7.8 \mathrm{~Hz}$ fundamental, about 2 to $3 \mathrm{pT}$, and the calculated and inferred strength of the global cerebral cortical of 2 to $3 \mathrm{pT}$. From this perspective the inclusion of diffusivity term (Equation 2) for the calculation of the 2 to $3 \mathrm{pT}$ we derived from the $\mu \mathrm{V}$ variations for the cerebral magnetic field is directly relevant.

If the human cerebral and the Schumann resonances are coupled then a mechanism implicitly connected to diffusivity through a magnetic medium would be expected if not required [50]. The involvement of the magnetic diffusivity term in deriving pT fields which when divided into the range of potential differences generated by Schumann resonances and cerebral cortical activity $\left(10^{-3} \mathrm{~V} \cdot \mathrm{m}^{-1}\right)$ approaches a velocity term for photons in a vacuum might help relate the chemistry, physics and astronomical variables shared by these "micro" and "macro" spherical wave conductors.

The representation of Schumann resonances within the human EEG profile in context of the normal power peak of $\sim 10-11 \mathrm{~Hz}$ or between the first and second harmonic of the Schumann series could have had adaptive value. If the cerebral power profile was bimodal or $8 \mathrm{~Hz}$ and $14 \mathrm{~Hz}$, the probability of more continuous congruence with the information contained within the Schumann carriers or wave guide would have increased. 
If our inferences are even partially valid concerning the coupling or equilibrium of the magnetic field and electric field intensities between the Schumann and human cerebral cortical sources, then near-continuous earth-ionosphere-brain interaction could dominate brain activity. It may be relevant that two major populations of human beings sometimes display a "splitting" power spectrum that drifts upwards toward $14 \mathrm{~Hz}$ and downwards towards $7 \mathrm{~Hz}$. The first is a subpopulation of human beings often diagnosed with terms such as schizophrenia. The second is the geriatric population, particularly when the cerebral histological changes emerge that are most frequently correlated with senile dementia.

The physical properties, complexity, and temporal congruence between real-time Schumann and brain values in our laboratory indicate that these considerations may have significant implications for the recondite influence of this planetary biofrequency source.

\section{THE IMPORTANCE OF HUMAN DENSITY FOR POTENTIAL CONVERGENCE BETWEEN GLOBAL SCHUMANN FREQUENCIES AND AGGREGATES OF HUMAN BRAINS.}

The phenomena of consciousness are often attributed to a relatively wide band of electrical fluctuations in the order of about $2 \mu \mathrm{V} \cdot \mathrm{Hz}^{-1}$ within the $40 \mathrm{~Hz}$ range. This is equivalent to about $25 \mathrm{~ms}$ which is within the range of the recursive, coherent waves of electroencephalographic potentials that move in a rostral to caudal direction over the cerebral hemispheres during the waking and rapid eye movement (REM) or dream states [15].

Assuming the average of $2 \cdot 10^{-6} \mathrm{~V}$ for this activity through the cerebral cortical tissue for which the intracellular resistivity is $\sim 2 \Omega \cdot \mathrm{m}$, the current would be $10^{-6} \mathrm{~A} \cdot \mathrm{m}^{-1}$ and when applied across the averaged linear distance of the cerebrum $\left(\sim 10^{-1} \mathrm{~m}\right)$, would be $10^{-7}$ A per human brain. For the present population of $\sim 7 \cdot 10^{9}$ human brains, the summed current is $7 \cdot 10^{2}$ $\mathrm{A}$ and when spread over the surface of the earth $\left(5 \cdot 10^{14} \mathrm{~m}^{2}\right)$ is about $1.4 \cdot 10^{-12} \mathrm{~A} \cdot \mathrm{m}^{-2}$. For comparison the mean of the air-earth current over land is about $2.0 \pm 0.3 \cdot 10^{-12} \mathrm{~A} \cdot \mathrm{m}^{-2}[51]$.

There are several independent indicators to discern this current density. According to Hill [52] a typical columnar resistance of $6 \cdot 10^{16} \Omega \cdot \mathrm{m}^{2}$ is evident up to $6 \mathrm{~km}$; the total columnar resistance is $\sim 8 \cdot 10^{16} \Omega \cdot \mathrm{m}^{2}$. The fair weather field at an altitude of $6 \mathrm{~km}$ is $2.2 \cdot 10^{5} \mathrm{~V}$ with a slight increase to $2.8 \cdot 10^{5} \mathrm{~V}$ within the domain of the electrosphere. Distributed over the surface of the earth, the current might be as high as $3.5 \cdot 10^{-12} \mathrm{~A} \cdot \mathrm{m}^{-2}$.

One increment of magnetic field intensity associated with QEEG activity within the human cerebrum has been shown to be $\sim 2 \cdot 10^{-12} \mathrm{~T}$. The product of magnetic flux density $\left(\mathrm{kg} \cdot \mathrm{A}^{-1} \cdot \mathrm{s}^{-2}\right)$, current density $\left(\mathrm{A} \cdot \mathrm{m}^{-2}\right)$ and magnetic diffusivity $\left(\mathrm{m}^{2} \cdot \mathrm{s}^{-1}\right)$, one potential process by which brain that share the earth's magnetic field or common current density might be associated, is $\mathrm{kg} \cdot \mathrm{s}^{-3}$ or $\mathrm{W} \cdot \mathrm{m}^{-2}$. Assuming the value of $10^{-12} \mathrm{~T}$ for either the functional magnetic field intensity of human cerebral activity or the first harmonic of the Schumann Resonance, $10^{-12} \mathrm{~A} \cdot \mathrm{m}^{-2}$ current density for either the earth-air value or the average for all of the brains of the human population, and $10^{6} \mathrm{~m}^{2} \cdot \mathrm{s}^{-1}$ (from $(\mu \cdot \rho)^{-1}$ where $\mu$ is magnetic permeability $\left(4 \pi \cdot 10^{-7} \mathrm{~N} \cdot \mathrm{A}^{-2}\right)$ and $\rho=$ the inverse of $2 \cdot \Omega \cdot \mathrm{m}$ or $0.5 \mathrm{~S}$, the radiant flux density would be $\sim 10^{-18} \mathrm{~W} \cdot \mathrm{m}^{-2}$.

The significance of this relatively small flux density becomes apparent when it is applied over the cross-sectional area of human cerebrum (cortex), about $10^{-2} \mathrm{~m}^{2}$. The resulting value is $10^{-20} \mathrm{~W}$ or $\mathrm{J} \cdot \mathrm{s}^{-1}$. This is the unit energy for a single action potential. Energy is the square root of the product of voltage-squared, the band width, and Coulombs $(\mathrm{A} \cdot \mathrm{s})^{2}$. For the typical voltage $(2 \mu \mathrm{V})$ of the QEEG across a band width of $100 \mathrm{~Hz}$ (where actual 
cerebral activity can range over $500 \mathrm{~Hz}$ ) and unit charge is $1.6 \cdot 10^{-19} \mathrm{~A} \cdot \mathrm{s}$, the square root of the $\mathrm{E}^{2}$ of $1 \cdot 10^{-40} \mathrm{~J}^{2}$ is about $1 \cdot 10^{-20} \mathrm{~J}[53]$. This relates the single energy associated with the action potential with the unit reflective of the operation of the whole cortices.

In addition the sharing of the solution for a velocity term that approaches $\mathrm{c}$ for the fundamental electric and magnetic fields for both the human brain and the earth-ionosphere cavity introduces the potential for quantum process coupled with photons emission and absorption. The time required for a photon with a velocity of $\mathrm{c}$ to traverse the plasma cell membrane $\left(10^{-8} \mathrm{~m}\right)$ of a neuron is about $10^{-16} \mathrm{~s}$ which is sufficient to converge with a single orbit of an electron thus allowing information from outside of the brain to influence brain matter and for brain matter to affect the photons emitted into space.

\section{CONCLUSIONS}

The quantitative mathematical solutions and data presented here strongly support previous and pioneering research that there is a direct connection between electromagnetic properties of the earth-ionosphere cavity and human cerebral activity. Generally speaking, the correlations observed between human quantitative encephalograms measured within our laboratory and the Schumann resonance measured here, in California and Italy suggests that the earth-ionosphere-brain connection is not bounded by local perturbations but exists across the entirety of the planet. The cerebral representations appear to be mediated through structures located within the caudal aspect of the brain, especially the temporal lobe.

While we have not systematically assessed structures outside of this general locus, previous experiments involving correlation and simulated geomagnetic activity suggest a strong involvement. The simultaneous recording of the Schumann resonances within an induction magnetometer and brain activity with a QEEG indicates that there are discrete temporal increments in which the Schumann resonances harmonize with the brain with phase shifts approaching 40 milliseconds.

\section{ACKNOWLEDGMENTS}

This manuscript is dedicated to the late Dr. H.L. König in appreciation for his efforts in confirming the presence of the Schumann resonance and for his insight into its similarity and possible relevance for the human brain. Thanks to Mr. John Carscallen and Mr. Renato Romero for their technical expertise during the construction of the preamplifier for "Herbert". The first author would personally like to thank Sam and Helen Saroka for their assistance in Herbert's construction.

\section{References}

[1] M. A. Persinger, Frontiers in Neuroscience 6 (2012), article 19.

[2] M. A. Persinger, International Letters of Chemistry, Physics and Astronomy 11 (2014) 24-32.

[3] A. Nickolaeno, M. Hayakawa, Schumann Resonances for Tyros. Springer, Tokyo, 2014.

[4] H. L. König, A. P. Krueger, S, Lang, W. Sonnig, Biologic Effects of Environmental Electromagnetism. Springer-Verlag, New York, 1981.

[5] N. Cherry, Natural Hazards 26 (2002) 279-331. 
[6] W. Hume-Rothery, Electrons, Atoms, Metals and Alloys 1963 Dover, N.Y.

[7] A. V. Streltsov, T. Guido, B. Tulegenov, J. Labenski, C.-L. Chang, Journal of Atmospheric and Solar-Terrestrial Physics 119 (2104) 110-115.

[8] S. M. Blinkov, I. I. Glezer, The Human Brain in Figures and Tables: A Quantitative Handbook. Plenum Press, New York, 1968.

[9] B. Pakkenberg, J. G. Gundersen, The Journal of Comparative Neurology 384 (1997) 312-320.

[10] D. C. Van Essen, H. A. Drury, The Journal of Neuroscience 17 (1997) 7079-7102.

[11] A. Bragin, C. L. Wilson, R J. Staba, M. Reddick, I. Fried, J. Engel, Annals of Neurology 52 (2002) 407-415.

[12] E. Niedermeyer, F. Lopes Da Silva, Electroencephalography: Basic Principles, Clinical Applications and Related Fields. Urban and Schwartzenbert, Baltimore, 1987.

[13] D. Kahn, H. F. Pace-Schott, J. A. Hobson, Neuroscience 78 (1997) 13-38.

[14] P. L. Nunez, Neocortical Dynamics and Human EEG Rhythms. Oxford University Press, New York, 1995.

[15] R. R. Llinas, D. Pare, Neuroscience 44 (1991) 521-535.

[16] T. Koenig, L. Prichep, L. Lehmann, D. V. Sosa, E. Braker, H. Kleinlogel, R. Ishehart, E. R. John, NeuroImage 16 (2002) 41-48.

[17] D. Lehmann, W. K. Strik, B. Henggeler, T. Koenig, M. Koukkou, International Journal of Psychophysiology 29 (1998) 1-11.

[18] G. Ryskin, New Journal of Physics 11 (1995) 0603015.

[19] J. P. Wilswo, J. P. Barach, J. A. Freeman, Science 208 (1980) 53-55.

[20] C. Pantev, S. Makeig, M. Joke, R. Galambos, S. Hampson, C. Gallen, Proceedings for the National Academy of Sciences U.S.A. 88 (1991) 8996-9000.

[21] R. Sandyk, Journal of Alternative and Complimentary Medicine 3 (1997) 365-386.

[22] P. A. Anninos, N. Tsagas, R. Sandyk, K. Derpapas, International Journal of Neuroscience 60 (1991) 141-171.

[23] J. C. Booth, S. A. Koren, M. A. Persinger, International Journal of Neuroscience 115 (2005) 1039-1065.

[24] D.D. Sentman, in Handbook of Atmospheric Electrodynamics Vol I, H. Volland (Editor). CRC Press, Boca Raton, 1995.

[25] G. Buzaski, Neuron 33 (2002) 325-340.

[26] M. F. Bear, Proceedings of the National Academy of Sciences, U.S.A. 93 (1996) 13453-13459.

[27] D. G. Amaral, R. Insausti, in G. Paxinos (Ed) The Human Nervous System, Academic Press, New York, 1990, pp. 711-754.

[28] D. R. Corson, D. Lorrain, Introduction to Electromagnetism and Waves. W. H. Freeman, San Francisco, 1962. 
[29] R. C. Burke, M. A. Persinger, NeuroQuantology 11 (2013) 1-7.

[30] M. A. Persinger, S. A. Koren, International Journal of Neuroscience 117 (2007) $157-175$.

[31] C-Y. T. Li, M-m. Poo, Y. Dan, Science 324(2009) 643-645.

[32] E. R. Graf, F. E. Cole in M. A. Persinger (ed) ELF and VLF Electromagnetic Field Effects, Plenum Press, N.Y., 1974.

[33] F. Aboitz, A. B. Scheibel, R. S. Fisher, E. Zaidel, Brain Research 2 (1992) 143-153.

[34] M. A. Persinger, Current Medicinal Chemistry 17 (2010) 3094-3098.

[35] P. Gloor, V. Salanova, A. Olivier, L. F. Quesney, Brain 116 (1993) 1249-1273.

[36] A. Alonso, R. Klink, Journal of Neurophysiology 70 (1993) 128-143.

[37] P. Gloor, The Temporal Lobe and Limbic System. Oxford, N.Y., 1997.

[38] H. E. Puthoff, Physical Review A General Physics 39 (1989) 2333-2342.

[39] M. Bordag, U. Mohideen, V. M. Mostepanenko, Physics Reports 353 (2001) 1-205.

[40] E. R. John, Mechanisms of Memory. New York: Academic Press, 1967.

[41] A. A. Minakov, A. P. Nikolaenko, L. M. Rabinovich, Radiofizika 35 (1992) 488-497.

[42] M. A. Persinger, International Letters of Chemistry, Physics and Astronomy 2 (2014) $15-21$.

[43] M. A. Persinger, International Letters of Chemistry, Physics and Astronomy 19 (2014) 181-190.

[44] Tomasz Borowski, International Letters of Chemistry, Physics and Astronomy 11 (2013) 44-53.

[45] S. N. Ahmed, S. A. Kamal, K. A. Siddiqui, S. A. Husain, M. Naeem, Kar University Journal of Science 5 (1997) 19024.

[46] M. A. Persinger, L. S. St-Pierre, International Journal of Geosciences 5 (2014) 450-452.

[47] L. C. Tu, J. Luo, G. T. Gilles, Reports on Progress in Physics 68 (2005) 1-110.

[48] B. T. Dotta, K. S. Saroka, M. A. Persinger, Neuroscience Letters 513 (2012) 72-80.

[49] A. Delorme, S. MaKeig, Journal of Neuroscience Methods 134 (2004) 9-21.

[50] M. A. Persinger, The Open Biology Journal 6 (2013) 8-13.

[51] H. Volland, Handbook of Atmospherics Volume I, CRC Press, Boca Baton (Fla), 1982, pp. 66.

[52] R. Hill, Pure and Applied Geophysics 84 (1971) 67-74.

[53] M. A. Persinger, C. F. Lavallee, Journal of Consciousness Studies 19 (2012) 128-153. 